\title{
Gradhiva
}

Revue d'anthropologie et d'histoire des arts

\section{La singularité fantôme. Hypnose, chasse aux spectres et cybernétique de la transmigration à Calcutta}

Spectral Singularity Hypnosis. Ghost Hunting and Cybernetics of

Transmigration in Calcutta

\section{Emmanuel Grimaud}

\section{OpenEdition \\ Journals}

Édition électronique

URL : http://journals.openedition.org/gradhiva/4011

DOI : 10.4000/gradhiva.4011

ISSN : 1760-849X

\section{Éditeur}

Musée du quai Branly Jacques Chirac

Édition imprimée

Date de publication : 29 mai 2019

Pagination : 50-83

ISBN : 978-2-35744-112-5

ISSN : 0764-8928

\section{Référence électronique}

Emmanuel Grimaud, « La singularité fantôme. Hypnose, chasse aux spectres et cybernétique de la transmigration à Calcutta », Gradhiva [En ligne], 29 | 2019, mis en ligne le 31 mars 2021, consulté le 02 avril 2021. URL : http://journals.openedition.org/gradhiva/4011; DOI : https://doi.org/10.4000/ gradhiva.4011 


\section{La singularité fantôme}

Hypnose, chasse aux spectres et cybernétique de la transmigration à Calcutta

par Emmanuel Grimaud

Cet article est un essai d'ethnographie dickienne, en hommage au dernier roman de Philip K. Dick, La Transmigration de Timothy Archer. À Calcutta, des patients explorent leurs «vies antérieures» dans le cabinet d'une hypnothérapeute, tandis que des chasseurs de fantômes enquêtent sur des maisons délabrées et tentent d'établir une communication avec des présences via des appareils électromagnétiques.

Alors que l'hypnotiseuse mène sa propre enquête sur la transmigration à travers les visions de ses patients, démêlant leurs identités troublantes et en quoi elles pourraient être liées, les chasseurs en quête de signaux font leurs propres expériences qui visent à surmonter la paréidolie pour entrer en contact avec des corps inconnus. Si les deux sont liés, ce n'est pas uniquement parce que réincarnation et hantise sont les deux composantes d'un même circuit (la cybernétique du samsâra), qui devient ici terrain d'expérience. C'est aussi parce que hypnose et chasse aux spectres visent toutes deux, bien que par des moyens expérimentaux différents, à s'approcher d'un point de catastrophe, d'un seuil critique de présence (appelé singularité fantôme), qui marque le jaillissement d'une vie spectrale foisonnante et imprévisible et d'où la matière humaine ressort à la fois entièrement questionnée et recomposée. 
1. Pour une analyse très fouillée du concile, voir Demiéville 1952. Pour une histoire des controverses et spéculations autour de la notion de soi entre bouddhistes et brahmanes, voir Hulin 2008a.

2. La Transmigration de Timothy Archer (Dick 1982) explore des angoisses chères à Dick: le fait de se sentir captif de l'univers d'un autre, d'être possédé par une entité sans le savoir ou de faire partie d'un grand circuit dont nous ne comprenons pas les règles. Les pages de L'Exégèse sur la

transmigration d'Archer, inspirées de la vie de son ami Jim Pike, son instructives: "Févriermars 74 : j’ai été possédé. But: réincarner Jim afin qu'il puisse continuer [...] à parler (à travers mes écrits, et pas seulement dans le livre sur Archer). " (Dick 2016 [1974-1982] : 80)

3. Sur l'art de convoquer des entités invisibles en TCl et d'interpréter les signaux, voir: Sconce 2000; Baudouin 2015 et 2018. Sur les phénomènes de voix électronique en particulier, voir: Raudive 1971; Banks 2001 ; Nees et Phillips 2014.

4. En sanskrit, littéralement, "ce qui circule"; désigne aujourd'hui le cycle des renaissances, des vies et des morts. Sur l'histoire de cette notion dans I'Inde brahmanique et bouddhique, voir Kapani 1993. Sur les réélaborations anciennes et modernes de la notion de dépôt karmique (traces d'expériences antérieures) et la possibilité d'accéder à la mémoire de ses vies antérieures (jātismaran), voir: Malamoud 2004 Hulin 1983 et 1985 ; Keyes et Valentine 1983; Guenzi et D'Intino 2016.
5. Bien après les prédictions de Bernal (1929) sur l'éthérisation de l'humain comme prochaine étape de l'évolution, la notion de singularité a fait l'objet d'amples spéculations chez les futurologues, écrivains de SF et idéologues pop du transhumanisme. qui prévoient dans un futur proche le franchissement d'un seuil critique où les machines dépasseront les humains en intelligence et au-delà duquel tout deviendra imprévisible. «Alors, l'histoire humaine aura atteint une sorte de singularité, une transition intellectuelle aussi

impénétrable que le nœud d'espace-temps au cœur d'un trou noir, et le monde passera bien au-delà

de notre compréhension." (Vinge 2019 [1983] :

140-143)

6. William James, dans La Volonté de croire (2005 [1897]), définit l'hypothèse vivante comme une véritable possibilité qui dispose à agir irrévocablement, et l'hypothèse morte comme une possibilité qui n'engage plus sur un mode viscéral ou qui a perdu sa capacité d'embrayage et ne résonne plus dans notre conscience.
Enfin, la conscience elle-même pourrait se dissoudre ou disparaître dans une humanité complètement éthérée, signant la perte de notre organisme si bien intégré, se transformant en masses d'atomes dans l'espace communiquant par rayonnement et se résolvant peut-être entièrement en lumière.

John Desmond Bernal, The World, the Flesh \& the Devil, 1929.

L'énigme a perduré bien après le fameux concile de Lhassa ${ }^{\mathbf{1}}$ (VIII ${ }^{\mathrm{e}}$ siècle), où bouddhistes et hindous s'affrontèrent au sujet de la transmigration en espérant apporter une réponse définitive. Savoir ce qui se décompose ici, survit, se transforme, se recycle ou se réagrège - de l'âme, des facultés, de la mémoire cellulaire, du code? - continue de stimuler un nombre incroyable d'enquêtes, d'expériences et de spéculations. Le débat ne concerne pas uniquement les réincarnationnistes, mais aussi la biologie (qui a encore bien du mal à dire de quelle mémoire génétique un embryon est exactement doté), et il déborde largement les espaces de spéculation autorisée de la théologie et de la science. Construit comme un hommage à La Transmigration de Timothy $A r c h e r^{2}$, le récit qui suit examine comment, à Calcutta, certains font leurs propres enquêtes en mettant les âmes errantes sur écoute, par des moyens qui relèvent de la transcommunication instrumentale $(\mathrm{TCl})^{3}$, mais pas uniquement. Le samsâra ${ }^{4}$ a sa propre cybernétique et le but de ces expériences est de s'approcher de son point de «singularité " (ou seuil critique ${ }^{5}$ ). À la différence de la singularité des prophètes du transhumanisme, placée dans le futur et qui marquerait le début explosif d'une vie artificielle sans précédent, il faut considérer la singularité fantôme comme étant située ici même, à l'ombre du vivant, au point extrême de pulvérisation des corps qui marque le début de la vie spectrale. De telles zones troubles d'expérience ont des mécanismes d'embrayage qui leur sont propres et Philip K. Dick est probablement le meilleur allié pour les penser. Parce que Dick fait tout pour cultiver le trouble ou le provoquer, je vais essayer de montrer quel intérêt nous avons à évoluer dans ces zones à la manière de Dick, c'est-à-dire non pas en s'accrochant au premier corpus de croyances toutes faites que l'on peut trouver, mais en accompagnant ceux qui en font un plan limite d'expérience. Par petits glissements successifs, il se pourrait bien que quiconque prenne aujourd'hui le mystère de la transmigration à bras-le-corps, quelle que soit sa posture de départ (bienveillance ou scepticisme), ne soit conduit à se recaler autrement dans ce monde-ci, dès lors que se dérobe en chemin le sol auquel sont amarrées ses certitudes les mieux établies. Et si la meilleure hypothèse, la plus vivante ${ }^{6}$, était celle qui permet de donner à l'invisible la cohérence d'un biotope?

\section{Paranormal Calcutta}

Soumen, jeune ingénieur indien de Calcutta, était à ce point intrigué par les phénomènes paranormaux qu'il avait créé sa propre société d'investigation à but non lucratif. II y avait embarqué plusieurs de ses amis, notamment Pranoy, spécialiste dans l'identification d'ADN pour la police locale, un musicien qui bricolait des tablas électroniques, un étudiant en communication et un juge à la cour de cassation. C'est ce dernier, Debashish, qui nous présenta Soumen. La société comptait désormais une quinzaine de membres qui se 


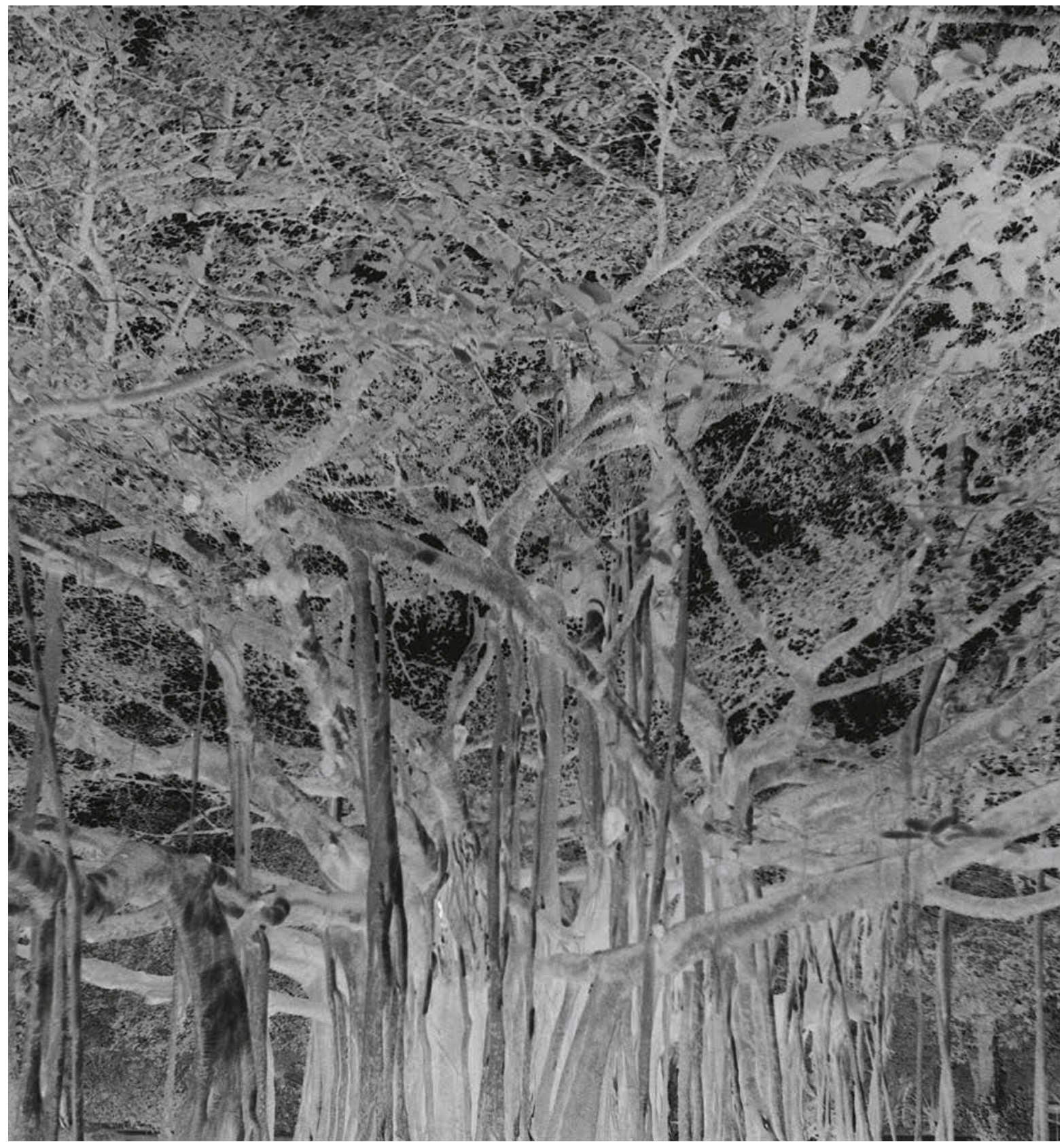

fig. 1

Banyan de Calcutta. C'est sous un

tel arbre que le sage Pippalada est

dit avoir composé le Garbhopanishad,

traité de l'Embryon, autour du Vle av. J.C.,

probablement l'une des premières

théories indiennes de la singularité

fantôme. Négatif sur support souple

en nitrate de cellulose. Août 1938.

Photo de Gabriel Monod-Herzen

() musée du quai Branly-Jacques Chirac. 


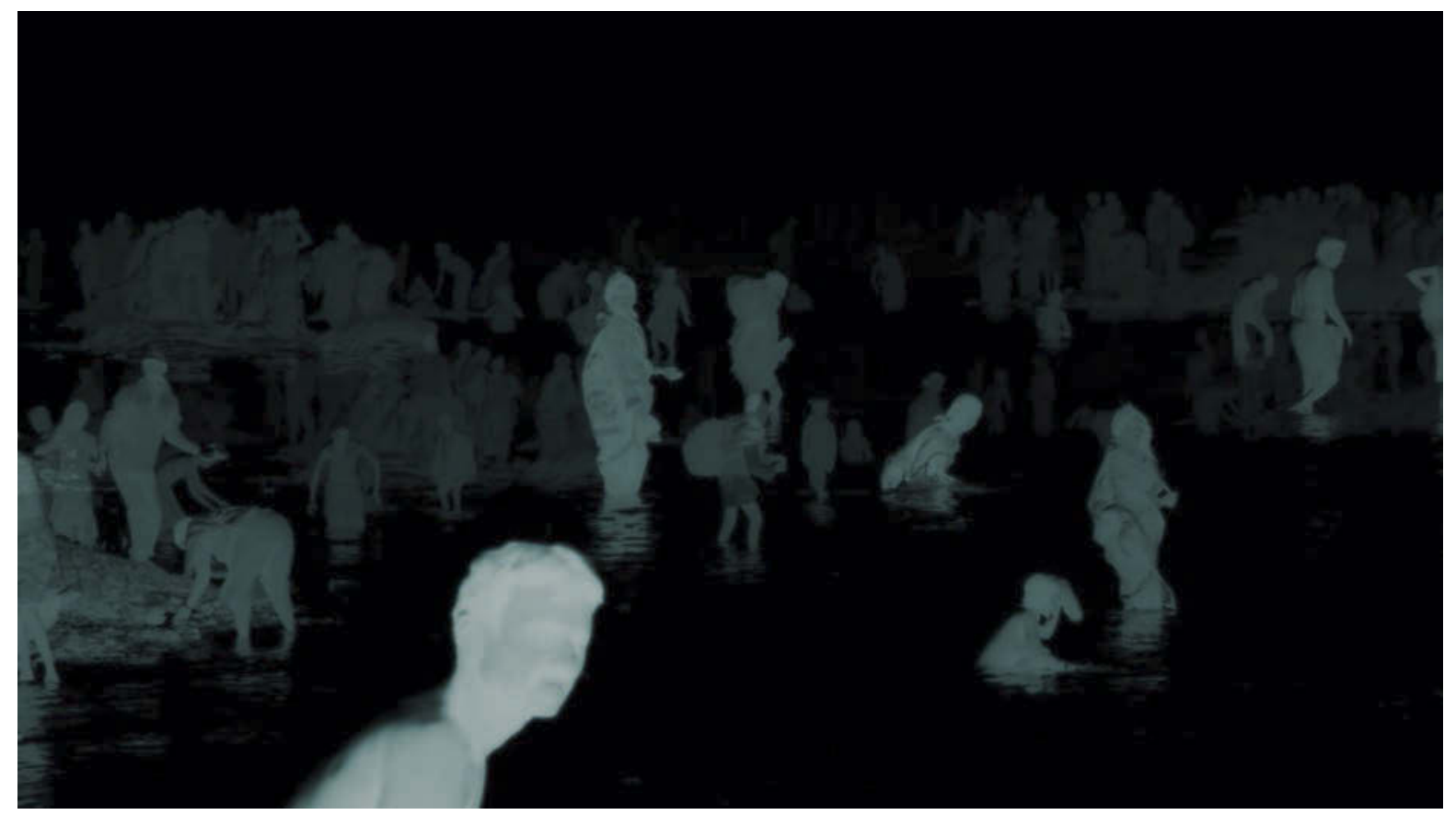


réunissaient régulièrement. Elle organisait des expéditions de ghost hunting, recevait des appels de gens s'estimant victimes de phénomènes étranges et se livrait à des séances de transcommunication instrumentale, via tout un tas d'appareils électroniques ou de radiofréquences, une passion largement internationalisée mais qui prenait à Calcutta, cette ancienne capitale de l'Empire britannique, une saveur particulière.

Debashish se prêta avec enthousiasme à une séance d'hypnose avec Trupti Jayin, une star de la past life regression et l'un des personnages principaux d'un film que nous tournions. Cette dernière avait pour l'occasion installé à Calcutta son cabinet baptisé Durga Transmigration Service. Le contrat était simple. II s'agissait de mettre Calcutta sous hypnose, ou du moins, un échantillon de personnes, pour aller dans leurs «vies antérieures» et voir... Trupti était habituée à donner des consultations individuelles et des workshops dans toute l'Inde. Elle trouvait aisément des patients dans les classes moyennes urbaines de plus en plus attirées par ce type de thérapie. Pour nous, l'expérience serait un moyen de saisir en temps réel ce qui se passe dans une séance d'hypnose et de collecter du matériau pour mieux comprendre cet état modifié de conscience. Pour elle, le film servirait à mieux analyser rétrospectivement les séances. Les émissions de téléréalité auxquelles elle avait participé étaient des dispositifs bien trop contraignants et sensationnalistes, tandis qu'une expérience anthropologique lui paraissait plus sérieuse et respectueuse de l'intimité requise pour le travail analytique. Le patient devait s'asseoir confortablement dans un fauteuil, après une brève conversation où il exposait à Trupti les raisons de sa venue. D'une voix suave, la thérapeute l'amenait ensuite à un état de relaxation suffisant pour le faire régresser dans son enfance, jusqu'à sa naissance et au-delà, dans ses précédentes «incarnations». Fouillant dans les tréfonds de sa mémoire, le patient prenait conscience des fardeaux hérités de ses vies passées (karma) pesant sur sa vie actuelle et traitait ses problèmes présents. Trupti était connue pour avoir à son actif une multitude de patients capables de témoigner de leur guérison. Ils avaient réussi, grâce à quelques séances de past life regression, à vaincre leurs traumatismes ou leurs phobies.

Debashish n'avait aucun problème apparent à traiter, en dehors de sa curiosité insatiable. Ce qui l'avait conduit ce jour-là chez Trupti Jayin était probablement une attirance pour les frontières de la réalité, celle-là même qui, à notre tour, nous a sans doute amenés à proposer à Trupti d'être le personnage principal du film. Elle nous avait invités à l'un de ses workshops à Bangalore, une thérapie de groupe, dont le moment le plus notable était moins le cours introductif rébarbatif qu'elle donnait sur la possibilité d'accéder à ses vies antérieures ou à des vies simultanées que l'un des exercices proposés: invité à déambuler dans un supermarché cosmique, chacun devait mettre dans un caddie tout ce qu'il désirait le plus dans la vie. Un couple qui souffrait de problèmes de fertilité, après avoir comme par magie trouvé un bébé dans les rayons de ce supermarché invisible, était ressorti de la séance revigoré. Le workshop était organisé dans un ashram tenu par un gourou new age qui faisait la promotion d'idées simples: «Soyez végétarien et faites de la méditation sous une pyramide. " Non loin de la salle où Trupti donnait son workshop, se trouvait un laboratoire rempli d'outils technologiques dernier cri

\section{ci-contre}

fig. 2

Fantômes prenant leur

bain. Film Black Hole,

Emmanuel Grimaud, 2019. 
7. Sur les origines de ce courant et les expériences de mesure du cerveau en méditation qui viennent à l'appui de la promotion du yoga, voir Tresch 2011.

8. "Ce qui donne leur densité aux humains, je n'aurai jamais la réponse. " Traduction de l'auteur. surfant sur la vague de la mindfulness ${ }^{7}$, où toute personne éprise de clairvoyance pouvait mesurer les bienfaits de la méditation sur son cerveau grâce à des électrodes ou en s'immergeant dans une piscine ultraviolette À quelques pas de là, un autre gourou local qui formait à l'hypnothérapie avait invité Trupti pour clore sa séance. II était connu pour guérir les gens de leur phobie des serpents, en leur permettant de toucher un de ces animaux préalablement mis sous hypnose, pour organiser des sessions de méditation collective autour des arbres (tree hugging), ou encore des séances de reconnexion à la Terre, en s'ensablant sur des plages. Mais cette fois, il n'y aurait aucune démonstration spectaculaire de sa part. Ce serait à Trupti de mettre quelqu'un sous hypnose en lui activant le «troisième œil» devant des apprentis ébahis, avant de reprendre son avion pour Mumbai.

Alors qu'à Mumbai les gens couraient après l'argent, à Calcutta, c'était une autre histoire. Et je m'étonne encore que Trupti ne nous ait jamais rien demandé en échange de sa participation au film. Là encore, c'est sans doute la curiosité qui l'a conduite à accepter les yeux fermés. Calcutta l'attirait par ailleurs pour une raison qu'elle n'arrivait pas vraiment à exprimer, outre le cliché que les Bengalis étaient des gens brillants qui avaient tout inventé. Trupti était convaincue qu'elle trouverait chez ce peuple exceptionnellement cultivé un autre type de patients, riches d'une vie intérieure surprenante. Elle installa son cabinet dans une vieille maison coloniale aujourd'hui transformée en pension, dont le propriétaire, Amritendu, accueillait volontiers des tournages pour financer la rénovation de l'héritage familial. II avait une passion pour les vieux meubles, les vases chinois antiques, les vieilles voitures. Tout ce qui avait moins de cinquante ans d'âge ne l'intéressait guère. Quand il apprit que des séances d'hypnose se dérouleraient dans une des chambres de la pension, il n'eut rien contre. Un bal d'hypnotisés le changerait du défilé de mannequins pour photo-tournages de mode qui se déroulait chaque jour dans son salon. Sa sœur était hypnothérapeute et il s'était livré avec elle à quelques séances de past life regression, au cours desquelles il était mort plusieurs fois et de manière atroce - notamment attaché à un pilier, torturé, les membres écartelés par un cheval. II n'avait pas voulu recommencer depuis. Sa sœur l'avait aussi aidé à se débarrasser de quelques fantômes récalcitrants qui hantaient la maison depuis la mort de son oncle. Nandu, l'intendant, fut le premier à en ressentir la présence, n'osant plus entrer dans l'une des chambres à l'étage. La sœur d'Amritendu fit un premier rituel pour chasser la présence non désirée et Amritendu appela ensuite un officiant rituel (pujari) pour achever le nettoyage. Mais il s'agissait d'un «bon fantôme » selon Nandu. Amritendu disait ne pas avoir de conviction particulière en la matière et faire ce genre de rituels uniquement pour rassurer l'entourage. Si Amritendu n'a jamais voulu que Trupti le mette sous hypnose, il n'avait aucun problème à ce qu'elle exerce sous son toit: "What makes us thick as human being ? I will never get the answer ${ }^{8}$ ", disait-il.

Le fantôme qui hantait la maison d'Amritendu venait probablement de la maison voisine abandonnée depuis longtemps, et avait séjourné quelque temps dans un arbre avant de se faufiler dans la chambre du haut. À Calcutta, les fantômes étaient partout, il suffisait de passer quelques heures avec Nandu pour saisir la banalité du phénomène. Ou bien d'allumer 


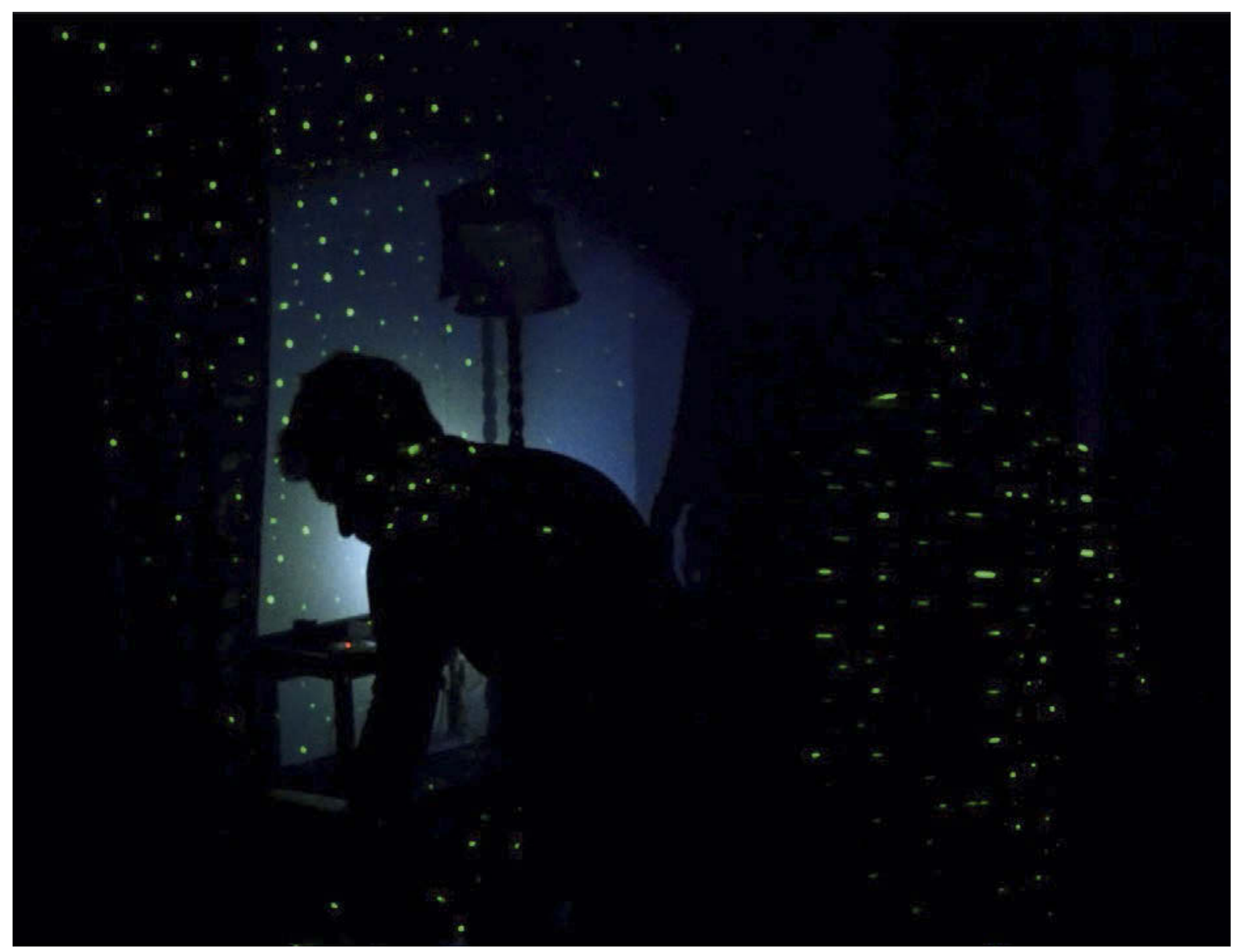

la radio et tomber sur Bhoot ${ }^{9} \mathrm{FM}$, une fréquence émettant depuis Dhaka qui nous rappelle qu'avant la partition du Bangladesh, le Bengale appartenait au même creuset culturel. La radio donne chaque semaine la parole à quiconque a un récit de fantôme à raconter et croule sous les témoignages. Tout au long du XIX ${ }^{e}$ siècle, Calcutta, ancienne capitale de l'Empire britannique, a été le principal foyer de la lutte pour l'indépendance de l'Inde et le lieu d'événements traumatiques. Elle compte aujourd'hui cinq millions d'habitants, et probablement le double si on inclut les multitudes de l'ombre qui sommeillent entre ses murs décrépis. Un véritable biotope d'entités à présence variable.

\section{Les âmes errantes ne sont pas ce que l'on croit}

La répartition sur Terre des spectres est loin d'être homogène. Contrairement à une idée reçue qui voudrait que nous baignions tous dans une même soupe invisible, il existe probablement des milieux plus denses que d'autres, des biotopes locaux aux frontières mouvantes, ou du moins des accès pluriels à la spectralité. Ce sont les contours de celle-ci que les ghost hunters s'attelaient à identifier, à grand renfort d'appareils électroniques. Quand ils débarquèrent dans la maison d'Amritendu pour y effectuer des mesures, ils essayèrent toutes les techniques possibles: détection au K2 $\mathrm{fig}=3$

Chasseurs de fantômes. Film Black Hole,

Emmanuel Grimaud, 2019

9. Bhuta signifie fantôme en hindi et bengali. 
10. La page Wikipedia "Fantômes de la culture bengalie " propose un inventaire très complet des spectres locaux, probablement une compilation de travaux des folkloristes. Mais devant une telle liste de créatures exotiques la réponse des ghost hunters fut lapidaire: "Ça n'existe pas, superstition."
Meter (un appareil aussi utilisé en géobiologie pour déterminer le niveau d'ondes d'un habitat), criblage de l'espace au laser, tentatives de communications à l'Echovox (un appareil qui brouille les champs radio, sur lequel je reviendrai plus loin). Leurs appareils photo et les caméras de surveillance installées dans chaque pièce laissaient bien transparaître quelques orbes de temps à autre, mais rien de suspect ou qu'on ne puisse attribuer aux défaillances de leur matériel. En revanche, le K2 Meter signalait un champ magnétique anormalement élevé, susceptible de provoquer des hallucinations et des problèmes intestinaux. Nandu, dans un premier temps, refusa de parler, de peur que les choses ne se sachent et fassent fuir les clients de la pension. II ne résista pas néanmoins à exprimer son sentiment de voir parfois une forme impromptue traverser les murs; impression disparue depuis l'intervention du pujari. Trupti s'était installée là quelques semaines, sans savoir qu'elle baignait dans un tel champ magnétique, et après avoir mis une vingtaine de personnes sous hypnose, elle reprit son avion pour Mumbai. Les ghost hunters, quant à eux, se devaient d'analyser plus en détail leur matériel, afin de se prononcer définitivement.

Leur mission chez Amritendu n'aurait été qu'un échec de plus dans leur traque habituelle, s'il n'y avait pas eu un lien entre leur recherche et les voyages dans les tréfonds de la mémoire collective auxquels les hypnotisés de Trupti se livraient quotidiennement. Je ne l'ai compris que bien plus tard. Pour l'heure, la maison était trop criblée de câbles électriques, sous terre et dans les airs, pour permettre une évaluation sérieuse par les ghost hunters. Une étude digne de ce nom supposait en effet un environnement non perturbé, ce qu'il était pratiquement impossible d'obtenir à Calcutta. Partout où ils allaient, les habitants faisaient les récits de fantômes les plus exubérants, mais ils se heurtaient à un champ électromagnétique chaotique qui rendait le diagnostic délicat, les opérateurs téléphoniques ayant sauvagement colonisé la ville, à coups d'antennes et de routeurs. S'enfoncer dans les campagnes en quête de forts abandonnés et de maisons délabrées était plus payant. En quelques années, ils avaient élargi leur périmètre d'investigation à la totalité du Bengale, en partant du principe que les spectres vivent en colonie tels des poissons et se déplacent à la vitesse de l'éclair partout où le milieu leur est propice.

De même que les pêcheurs ont de grands débats sur le type de mouches qu'ils utilisent, le bon geste à effectuer ou encore les habitudes de vie des poissons, les ghost hunters vouent un véritable culte à la technologie et passent leur temps à éplucher les catalogues d'appareils, tout en faisant des hypothèses sur la vie des spectres. Une conversation avec eux dérive inévitablement vers des questions de physique quantique sur les ondes et les champs magnétiques, la nature du hasard et les probabilités, abordées d'une manière singulière qui laisserait peut-être des physiciens perplexes, mais qui doit être examinée sérieusement car, après tout, s'il y a une soupe cosmique, nous sommes tous concernés. Une chose est sûre, les ghost hunters ne se contentent pas de cultiver le répertoire des fantômes des folkloristes ${ }^{10}$. Ils le jugent même totalement absurde, ce qui ne les empêche pas de prendre un malin plaisir à inventer des tas d'appareillages très techniques pour détecter des effets de présence. L'hypothèse à la fois la plus juste et la plus vivante, 
selon eux, c'est que les âmes errantes ne sont pas ce que l'on croit. Si personne ne peut vraiment dire ici de quoi elles sont faites, c'est que pour l'enquête il vaut mieux partir du postulat qu'elles ne ressemblent à rien de connu, et j'insiste sur ce point: ni humain, ni animal, ni germe ${ }^{\mathbf{1 1}}$, elles seraient plus proches de concrétions quantiques dont le mystère ne peut s'éclaircir qu'à l'échelle atomique. Et encore, rien n'est moins sûr.

La curiosité des ghost hunters pour ce qui se joue aux limites de la science dépasse largement le commerce des âmes errantes florissant de Calcutta. Ils y participent pour rendre service, sans jamais exiger de paiement en retour. II arrive qu'ils reçoivent des appels de gens désireux d'identifier des présences chez eux et ils s'y rendent volontiers, mais ils restent très prudents dans leurs diagnostics, qui se soldent à quatre-vingts pour cent par une explication qu'ils jugent «scientifiquement rationnelle». Quand l'enquête répond à la demande d'un commanditaire, comme ce fut le cas dans la maison d'Amritendu, ils n'interviennent que dans la phase de détection. Ce n'est pas à eux d'apaiser des âmes le plus souvent mortes prématurément, de façon traumatique, ou bien restées trop attachées à la vie en raison d'un amour inassouvi. Les exorcistes et les pujaris sont bien mieux équipés qu'eux pour cela et purifient les lieux à coups de formules (mantra) et de rituels. Les ghost hunters se prononcent uniquement sur le type de nourriture appréciée par l'âme errante, indiquant au pujari ses friandises favorites à inclure dans le menu rituel, une information importante qu'ils obtiennent généralement au terme d'une longue séance de questions-réponses avec ladite âme. Si celle-ci tient à hanter un lieu, c'est qu'elle réclame réparation. Le but de l'opération est alors de l'identifier pour qu'elle s'en aille en toute sérénité et se réincarne correctement. D'ailleurs, les rituels funéraires (tarpan) ne visent qu'à empêcher le retour des âmes récalcitrantes et leur assurer une réincarnation en toute fluidité. Fantômes et réincarnés s'attroupent aux deux extrémités d'un même circuit spectral qui échappe aux vivants. Un circuit paradoxal, jugé hors sol par ceux qui doutent, adhérant aux tréfonds du réel pour les autres. C'est par passion de l'inconnu et parce qu'il est persuadé qu'à cette frontière-là se joue autre chose que de vulgaires bugs dans le circuit, que Soumen a monté sa société paranormale: non pas pour se faire de l'argent sur le dos de ses concitoyens, mais pour repousser les limites de la technique en se frottant aux marges de la perception, à ses angles morts. Soumen, comme Dick, est convaincu que des corps inconnus dans le champ de la réalité peuplent l'invisible et qu'il faut en comprendre la cybernétique cachée. D'où la nécessité d'enquêter et de continuer la traque jusqu'à l'obtention de l'information manquante.

\section{Aux frontières de la mémoire}

Trupti Jayin avait probablement le même goût pour l'inconnu, toutefois, formée en psychiatrie, elle y accédait par le cerveau humain. En marge de ses consultations, elle échangeait avec des personnalités éminentes du champ des reincarnation studies et essayait d'obtenir des données plus précises sur la transmigration. Gourous, clairvoyants ou encore chercheurs en parapsychologie (dans la lignée d'lan Stevenson): tout interlocuteur lui permettant de peaufiner sa vision de la chaîne de traitement des âmes était bon à prendre. Un jour, Amritendu lui rendit visite avec le pujari qui l'avait aidé
11. Le médecin ayurvédique bengali Surendranath Goswami interpréta les spectres comme des germes, des parasites animaux et végétaux, relisant en termes bactériologiques

la flore spectrale

de la bhutobidya

("connaissances des fantômes ") des traités sanskrits (1911 et 1914). Pour s'immerger dans le bouillon spéculatif de l'époque coloniale d'où la médecine ayurvédique est sortie repensée, voir Mukharji 2016. 


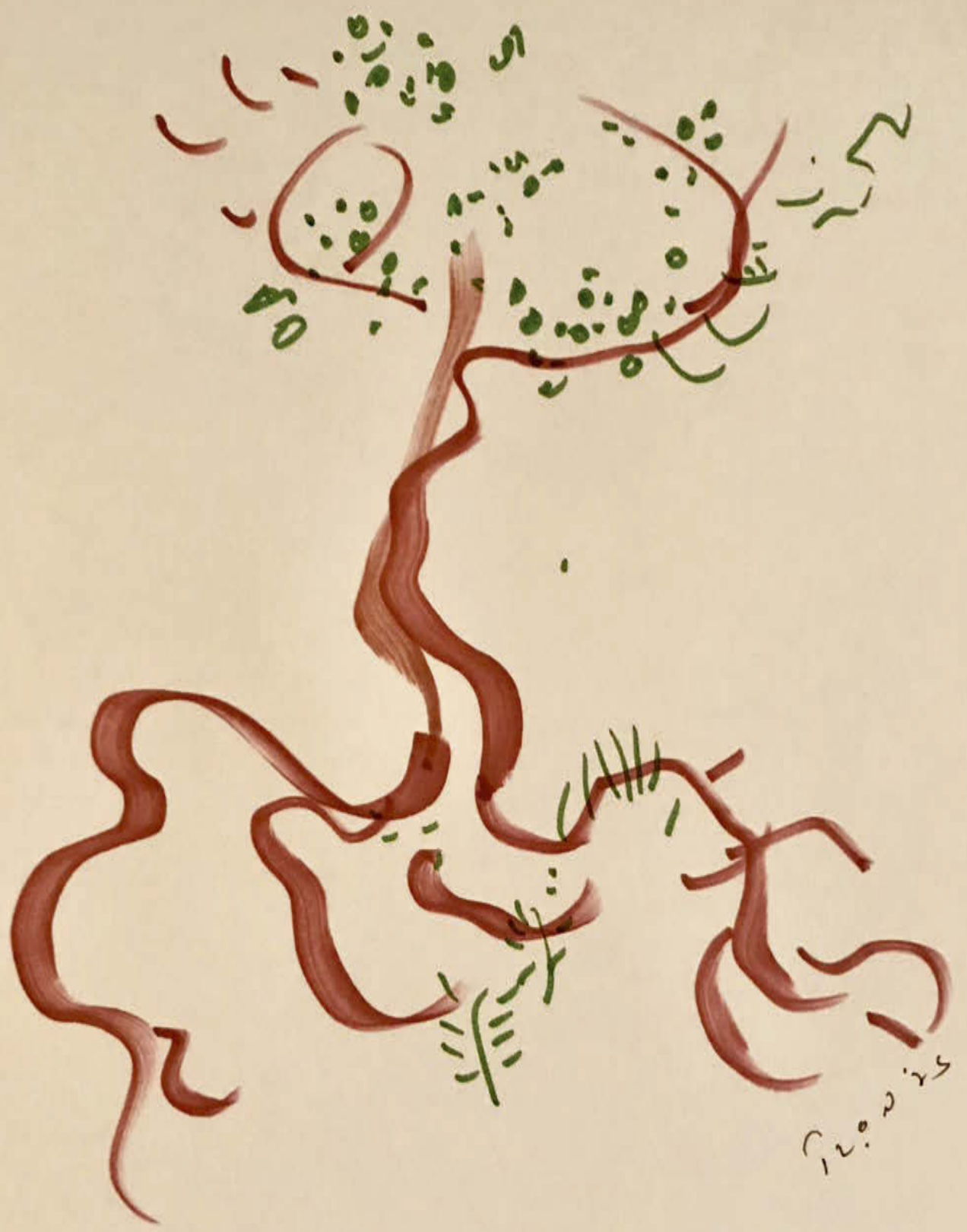


à débarrasser sa maison de ses fantômes. Trupti l'attaqua alors frontalement. Le débat portait sur le laps de temps où les âmes sont supposées être en errance, juste après la mort, avant de se réincarner dans un autre corps. D'après le pujari, cette errance dure deux ans. Les rituels (tarpan) destinés à nourrir l'âme du défunt par des offrandes (pindal) et à lui garantir une bonne réincarnation en lui constituant un corps astral doivent s'échelonner sur toute cette durée. Par les offrandes, on nourrit à la fois un défunt particulier et ses ancêtres, mais aussi ceux de toutes les âmes invisibles, à commencer par les sâdhus restés célibataires et sans progéniture, qui en ont donc bien besoin. Pour Trupti, une errance aussi longue est impossible, la «recherche scientifique » attestant, dit-elle, des cas d'enfants morts brutalement réincarnés au bout de trois mois. Le pujari lui répondit qu'il n'y avait rien d'impossible mais que «ce n'est pas parce qu'on a l'impression que son fils ressemble à son grand-père qu'il en est pour autant la réincarnation». Et poussé dans ses derniers retranchements, il rappela à Trupti que personne ne pouvait avoir de théorie définitive sur un mécanisme qui échapperait toujours à l'entendement humain et que les rituels, loin d'être obligatoires d'après les textes, étaient surtout destinés à rassurer les vivants.

Dans les spéculations sur la transmigration, le moment d'errance de l'âme juste après la mort constitue une zone trouble, un genre de trou noir dans la vie des corps qui fait toujours débat, pouvant varier de quelques jours à plusieurs mois, et à l'issue incertaine. Il est possible de bifurquer, de revenir hanter les vivants ou de se réincarner dans un autre corps, sans que l'on puisse savoir lequel, et ceci suivant une logique qui mérite investigation. Existait chez Trupti le désir de simuler expérimentalement cet état quand elle mettait les gens sous hypnose, les immergeant délibérément dans un trou noir où ils faisaient l'expérience de leur propre transmigration. Ce que j'ai dit précédemment des ghost hunters qui participaient à la chaîne locale de traitement des âmes errantes, tout en poursuivant une quête plus large aux limites du connu, s'applique aussi à Trupti. Certes, sa technique de guérison semblait avoir fait ses preuves, mais elle ne cessait de s'interroger. Chaque patient était l'occasion de progresser dans son enquête sur les frontières de la mémoire. Si elle se laissait volontiers impressionner par des cas «scientifiques $^{\mathbf{1 2}}$ » de vraies réincarnations, elle était aussi capable, envers les plus sceptiques, de faire sienne la formule de Tertullien, le théologien de Carthage, à propos de la résurrection: «Il faut y croire, parce que c'est impossible» (cité dans Dick 1982: 361).

Il n'est pas nécessaire de croire à la réincarnation pour s'adonner à une séance d'hypnose, et nombreux sont les patients sans conviction particulière sur leurs éventuelles vies antérieures qui arrivent là par curiosité. Aux plus sceptiques, Trupti disait que l'hypnose leur permettrait de se connecter à la partie étrange d'eux-mêmes, celle qui n'a jamais la parole. Parfois elle devait passer de longues minutes à convaincre quelqu'un de fermer les yeux. Et généralement, le doute ne compromettait pas la possibilité de voir quelque chose se produire. Plus le doute initial était grand, plus les chances de conversion étaient importantes. Consultant Trupti pour la première fois, M. Som avait bien pris soin de préciser qu'il ne croyait pas à l'idée de vies antérieures et qu'il n'avait foi qu'en l'astrophysique. S'il avait été torturé,
12. Dans La Transmigration...,Dick

examine tous les rapports de croyance possibles au sein du réincarnationnisme (disons du résurrectionnisme, sa version chrétienne). Autour d'Archer, il y a ceux qui pensent que la transmigration n'existe pas, ceux qui sont prêts à y croire si on leur en apporte la preuve, ceux qui sont dans l'incertitude totale et s'accommodent de ne pas savoir, et enfin ceux qui pensent qu'il y a bien transmigration mais qu'il faut s'attendre à ce qu'elle fonctionne de façon radicalement différente de ce que l'on croit. Ces quatre attitudes se retrouvent chez les personnages de notre récit, et peuvent même parfois coexister chez Trupti Jayin, selon ses interlocuteurs.

\footnotetext{
ci-contre

fig. 4

La singularité fantôme, dessin sous hypnose, Hiran Mitra, 2018.
} 

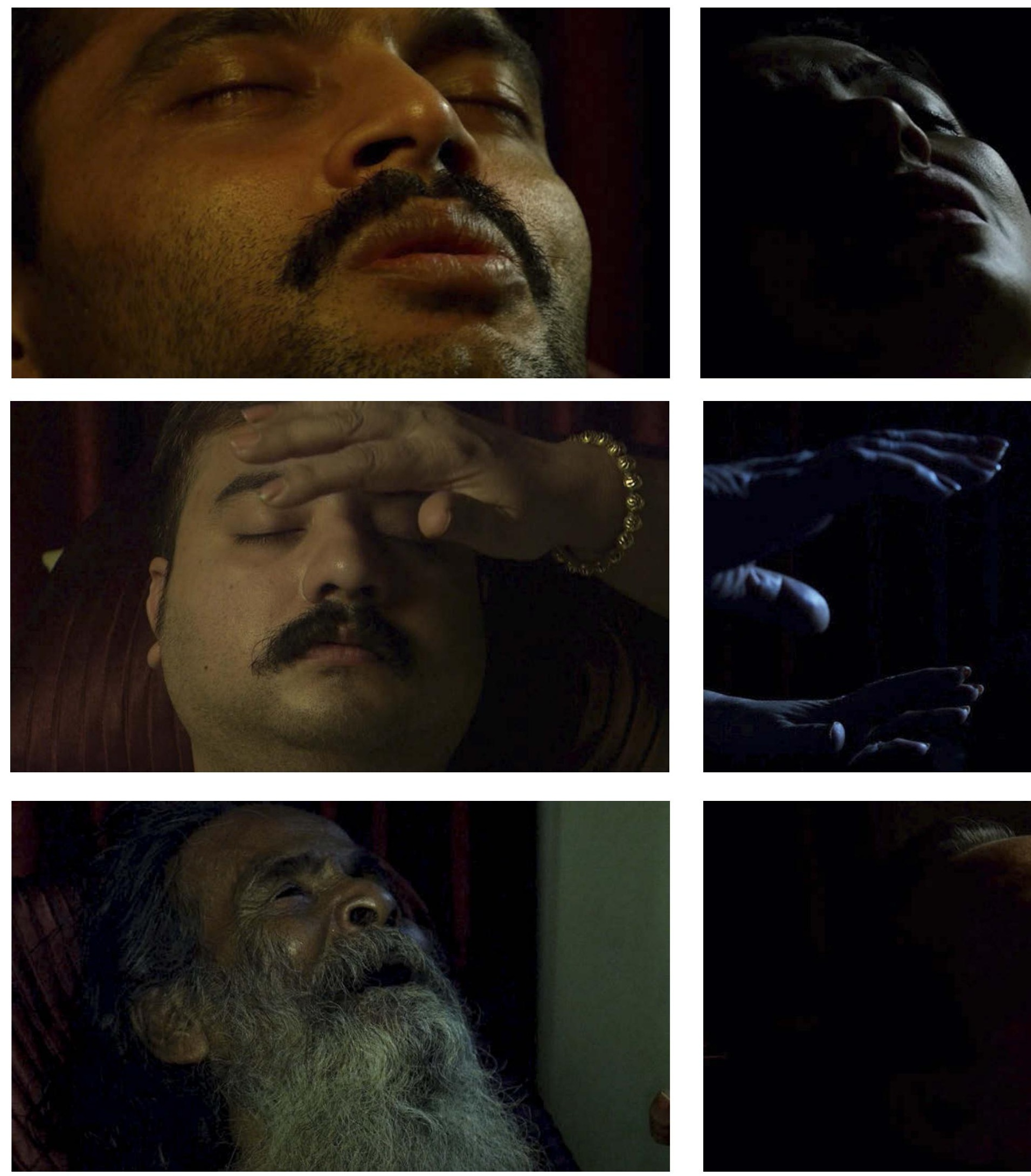

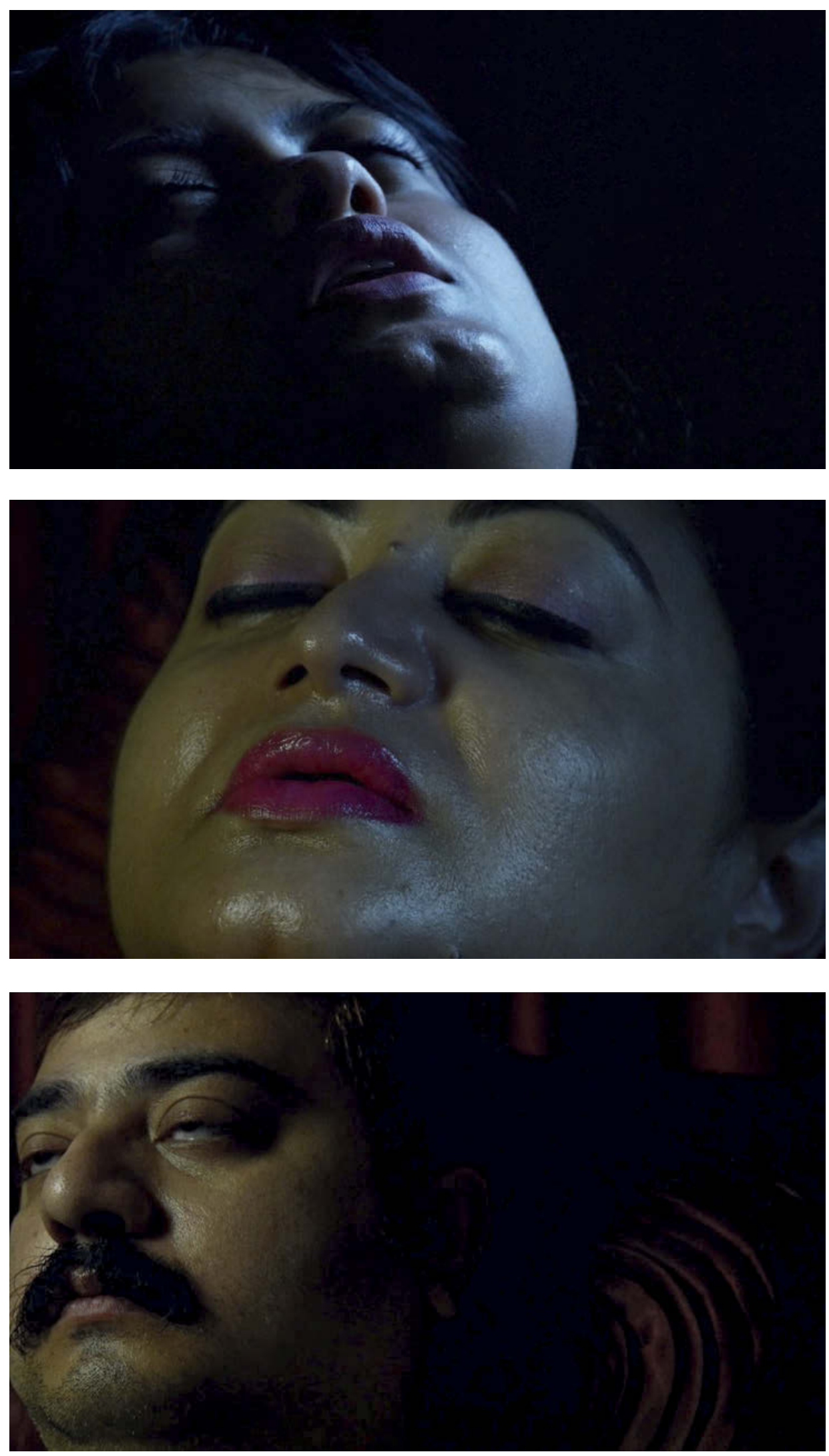
double page
précédente

fig. 5

Visages d'hypnotisés. Film Black Hole, Emmanuel Grimaud, 2019. En haut, de gauche à droite: Nandu, Sanchita, Nandini.

Milieu: Debashish, Kalyan, Satarupa.

En bas: Kanai Das Baul, Mr. Som, Debashish. c'était en raison de son action politique. Ce professeur de physique, capable de citer dans la même phrase Stephen Hawkings et Vivekananda, avait été pris par erreur pour un militant naxalite dans les années 1970. II subit d'atroces tortures policières en prison, avant d'être libéré. Le naxalite n'était pas lui, mais son frère. Pour Trupti, il devait bien y avoir quelque chose en fouillant un peu. Mais M. Som fut incapable de partir sous hypnose. Le physicien se retrouvait systématiquement bloqué dans un immense trou noir, d'où il lui était impossible de sortir, sauf à quelques moments fugaces où il percevait un éclair de lumière et des ombres végétales.

II n'y avait probablement aucun lien entre ce traumatisme toujours vivace chez M. Som et le supplice subi par Amritendu, mort écartelé dans une vie antérieure, que nous avons raconté précédemment. Jamais personne n'aurait eu l'idée de faire le lien, ni Trupti ni nous-mêmes, alors que nous explorions toutes les connexions possibles entre les récits des hypnotisés sur la table de montage. Imaginez: deux personnes font des récits de torture, pour l'un, probablement imaginaires sous hypnose; pour l'autre, issus de sa vie passée, avant de se retrouver dans un trou noir. Quelques jours plus tard, des ghost hunters débarquent chez Amritendu et concluent à un champ magnétique un peu trop élevé! Vouloir lier ces événements dans un circuit de cause à effet aurait été une folie. Et même s'il y a des moments où la folie, selon un personnage de La Transmigration de Timothy Archer, est "comme les petits poissons, se déplace en foule, se reproduit à de multiples exemplaires et se déploie d'un bout à l'autre du paysage » (Dick 1982: 341), il n'y avait là que des coïncidences malheureuses. Aucun signe apparent d'une anomalie dans le champ de la réalité. Aucun indice permettant d'en savoir plus sur une quelconque perturbation dans la chaîne de traitement des âmes. Mais pour Trupti, qui menait son enquête en entrant dans le cerveau des gens, il en était tout autrement. II s'agissait de signaux faibles en attente d'une connexion véritable et il fallait patiemment les inventorier en attendant que le lien ne saute aux yeux. Le film que nous tournions constituerait l'archive de cette recherche en temps réel. II fallait que l'on trouve des connexions, sur la table de montage.

Après M. Som, d'autres patients se succédèrent dans le cabinet de Trupti. Certains avaient bien du mal à lâcher prise. D'autres entraient en hypnose plus facilement et parfois, plus rarement, avant même que Trupti les ait invités à se relaxer. II suffit de quelques patients pour comprendre qu'il est impossible de prévoir, les plus créatifs n'étant pas forcément ceux que l'on croit. Et à Calcutta, nous allions de surprise en surprise. Trupti savait relaxer les gens, leur donner le sentiment de se délester de leur corps ne serait-ce qu'un instant, en les faisant passer (mentalement) par la fenêtre, survoler Calcutta comme un oiseau, transpercer les nuages pour flotter ensuite dans l'immensité du cosmos. Le patient pouvait se retrouver propulsé très loin avec un sentiment unique de légèreté, hors sol. Après ce délestage qui pouvait durer plus ou moins longtemps, le mental devait se poser quelque part, atterrir dans un corps: "Où es-tu? Quel corps as-tu? Décris-moi ce que tu vois..." Puis lorsque les détails commençaient à surgir, la texture d'un sol, une manière de poser le pied quelque part, une couleur de peau, le pli d'un habit, elle cherchait à en savoir plus sur le lieu, la date, et agissait comme une machiniste, indiquant au patient quel travelling opérer dans la scène, où faire 
un gros plan. Et lorsque par mégarde, l'hypnotisé était bloqué quelque part, elle faisait de grands sauts dans le temps, à coup de rewind et forward, pour que le film avance, celui du patient, celui qu'elle imaginait qu'il se faisait, et le nôtre, qui serait une synthèse de tous ces films.

L'hypnose a tout d'un cinéma mental, dont le patient est à la fois l'acteur, le réalisateur et le spectateur. Les yeux vibrent, parfois révulsés, mais rien de ce qui ce qui se passe à l'intérieur n'est véritablement accessible quand on le filme. La mémoire cherche, elle balaye peut-être des catalogues d'images, elle effectue probablement des milliards de connexions à la minute. Pour la première fois dans ma vie d'anthropologue, je ressentais d'aussi près ce qui se passe dans la tête d'un autre, un feu d'artifice neuronal intense que seules trahissent les vibrations des paupières. II fallait se contenter des indices de l'hypnotisé, en position de commentateur de ses propres actions dans le flot d'images mentales qui le traversait, sans pouvoir aller plus loin dans l'exploration de ce paradoxe humain: plus l'individu s'adonne, comme c'est le cas dans l'hypnose, à une expérience hors sol, plus il a le sentiment d'entrer en communion avec les profondeurs de lui-même, avec le mouvement secret de la Terre ou du cosmos. Ou pour le dire simplement: plus il lâche prise, plus il se connecte. Outre le sentiment océanique qu'il lui est donné ici d'éprouver, l'individu fait face à l'étrangeté de son propre cerveau. Et Trupti n'avait pas d'autre objectif que de stimuler cette crypto-activité intérieure, sans jamais juger ni interpréter; que le patient se confronte à ses propres énigmes, que ses images mentales s'épanouissent à la surface de son écran mental avec le plus de richesse possible, fourmillantes de détails étranges sur lesquels rebondir. Elle n'était qu'une médiatrice permettant à l'estrangemental de chacun de s'exprimer au grand jour. Au patient ensuite de faire sa propre enquête.

Il arrivait qu'une date, un lieu, un nom obtenu sous hypnose le pousse à faire ses propres recherches, qu'il croie ou non à la réincarnation. Comment expliquer que Nandini, par exemple, venue consulter Trupti pour une phobie du noir, se soit vue sous hypnose auteure d'un attentat au début du xxe siècle contre un général anglais avant de finir exécutée? Elle prit conscience plus tard que son grand-père était un proche de Khudiram Bose, jeune indépendantiste bengali, aujourd'hui salué en martyr de l'indépendance et mort exécuté pour ses actes de résistance. Nandini elle-même ne put s'empêcher de faire le lien. Elle n'alla pas jusqu'à penser qu'elle était la réincarnation de Khudiram, mais il y avait quelque chose d'apparenté entre sa vision et la vie de l'indépendantiste qui la laissait songeuse. Ses images sous hypnose avaient été d'une rare précision: la bombe explosant sous la calèche du général, les rues de Calcutta qu'elle avait traversées pour arriver au lieu-dit, le visage de ses collaborateurs, y compris celui de son professeur qu'elle admirait tant et qui avait fomenté l'opération en cachette dans une salle de l'université. La scène avait été trop vivante et pleine de détails plausibles pour n'être que le fruit de son imagination. Pour Trupti, il y avait plusieurs explications possibles, cependant l'idée que la mémoire d'un autre, et notamment d'un ancêtre aussi proche qu'un grand-parent, ait trouvé ici l'occasion de s'exprimer n'était pas à écarter. Après tout, que savons-nous de ce qui se transmet dans les tréfonds de nos gènes ${ }^{13}$ ?
13. Sur les neurosciences face à l'hypermnésie ou "mémoire autobiographique hautement supérieure» (MAHS), voir Luria 1995 . McGaugh 2014. 
En franchissant la porte de Durga Transmigration Service, les patients participaient à un grand sondage effectué dans la mémoire collective de Calcutta. Tout ce qui, sous hypnose, se ravivait dans la mémoire de l'un, appartenait peut-être aux autres. Mais il fallait l'éprouver expérimentalement et ne pas se contenter de la grande abstraction commode, toujours aussi difficile à ancrer, de la mémoire collective. Trupti tarda à se rendre compte qu'elle mettait le doigt sur autre chose que des mémoires individuelles ou une psyché singulière. Elle avait bien le pressentiment que des liens plus profonds existaient entre les mémoires de chacun, mais elle était trop souvent épuisée par l'intensité des séances pour les décortiquer à chaud et elle comptait sur le film, tel un dispositif médiumnique, pour les lui révéler. "Vous verrez au montage, nous avait-elle dit, il faut tout transcrire et voir par mots clés si des liens se font."Vingt personnes avaient été mises sous hypnose, donnant lieu à huit cents pages de transcription. Et six mois passèrent à explorer tous les liens possibles entre les récits des hypnotisés, en attendant la révélation...

C'est ainsi que certains indices commencèrent à piquer notre curiosité, car sans qu'on puisse rigoureusement le démontrer, un récit commun prenait forme, à condition qu'on examine chacune des scènes vécues sous hypnose à l'aune de son potentiel de liaison avec d'autres. En effet, chaque scène ou intrigue vécue par un individu était susceptible de trouver un prolongement ou sa solution en dehors d'elle-même, dans le récit d'un autre, formant ce qu'on décida d'appeler au montage une bande passante. Une telle bande était constituée des synchronicités possibles entre les récits des uns et des autres, des connexions plus ou moins fortes, des possibilités plus ou moins lâches de congruence. Trupti l'avait bien pressenti, pour qui la solution au traumatisme de l'un pourrait bien résider dans l'hypnose d'un autre, une hypothèse de travail que lui inspira l'échec de l'hypnose de M. Som. Venu avec un beau traumatisme, il n'avait jamais vraiment embrayé dans une vision susceptible de le déplacer. En revanche, il sortit de son trou noir brièvement, pour décrire sa relation fusionnelle avec une étudiante. Et le portrait de celle-ci était assez congruent avec celui que Nandini avait donné d'elle-même.

Ce n'est qu'au montage, à force de tenter de synchroniser les récits entre eux, que la bande passante de l'Attentat contre le général anglais prit tout son sens. D'autres hypnotisés semblaient naturellement y trouver leur place. Kalyan, cet ancien séducteur de la bonne société de Calcutta, se livra à l'expérience. Aussi peu convaincu que M. Som par l'idée qu'on puisse accéder à ses vies antérieures, il était cependant intrigué par le fait que «des données puissent être gardées en mémoire quelque part » dans "ce dispositif électronique qui nous sert de cerveau ". Incapable de fermer les yeux, il les avait gardés grand ouverts, puis, partageant l'intuition qu'il pourrait bien avoir été une femme dans une vie antérieure, il décrivit avec moult détails comment son frère, luttant lui aussi contre les Anglais, avait passé sa vie à tenter d'échapper à la police. M. Som aurait bien pu être cet homme, en plus d'avoir été le professeur de Nandini. Quant à Nandu, I'homme de maison d'Amritendu, sa vision la plus étonnante fut un accident de voiture, d'où il sortit les jambes coupées. Un épisode que Nandini explora à sa façon: avant de commettre 
son attentat contre le général, elle l'avait suivi, et à deux doigts de se faire attraper par la police, elle avait été sauvée par ses complices qui l'avaient embarquée à bord d'une voiture qui subit peu de temps après un accident similaire. Soumee, une neurobiologiste qui se prêta à l'exercice, elle aussi sans grande conviction au départ, se transporta à son tour en plein combat pour l'indépendance de l'Inde, à l'époque d'une figure célèbre de la Renaissance bengalie, Raja Ram Mohan Roy. Elle se sentait être lui, haranguer les foules et les encourager à lutter lors d'un meeting: "You cannot be ruled ", leur dit-elle. Sa voix avait complètement changé de texture et elle ne se souvint pas de grand-chose une fois la séance terminée. Nandini décrivit un meeting similaire où elle se sentait galvanisée avant d'accomplir sa mission, qui se termina par son exécution. La bande passante commençait donc avec le trou noir de M. Som et s'achevait par un autre trou noir, celui dans lequel Nandini avait été plongée, une fois mise à mort par son bourreau. Entre les deux, n'importe qui pouvait avoir été pris dans le film d'un autre comme acteur, spectateur ou réalisateur.

Voilà qui rappelle un principe de composition exploré par Philip K. Dick dans son roman L'CEil dans le ciel (1957). Suite à l'explosion dans le bévatron d'un déflecteur de faisceau protonique, sept visiteurs et leur guide se retrouvaient gisant au sol, inconscients, et se mettaient à créer un monde de pacotille à l'image de leur psyché, où les autres étaient embarqués sans le savoir et dont ils ne pouvaient plus sortir, jusqu'à ce que le sentiment d'une réalité une et indivisible se délite entièrement chez le lecteur. Bien évidemment, aucun artifice de montage n'aurait pu permettre de jouer ainsi avec le matériau dont nous disposions. En revanche, il m'a semblé instructif qu'un processus thérapeutique visant à sortir le patient d'un trou noir (ou dans le cas de Nandini d'une phobie du noir) s'achève par une nouvelle plongée dans un autre trou obscur, ailleurs et autrement. S'il était certes aventureux de concevoir que Nandini ait hérité du trou noir de M. Som, par une sorte de délégation ou de transfert de karma, postuler qu'entre les deux une parenté existe était-il totalement absurde?

\section{Soupe d'ondes}

Tout comme Trupti, les ghost hunters avaient besoin du noir. Tandis qu'elle multipliait les consultations, ils poursuivaient leur traque de nuit, sans autre objectif que de débusquer de la présence quelque part, à l'aide d'un dispositif extrêmement méthodique et appareillé. Suivant des fiches très détaillées avec des critères à toutes les étapes du processus, chaque lieu fait l'objet de mesures et d'un examen protocolaire. II y a même chez eux un culte de l'administration de la preuve, une méthode pour identifier des anomalies perceptives (ou ce que William James appelait le «résidu non classé»), des questionnaires précis à adresser aux présences pressenties, ainsi que des procédures de contrôle des interférences possibles de l'expérimentateur ou de ses extrapolations dues à la paréidolie (notion sur laquelle on reviendra plus loin). Mais tout commence dans l'obscurité, condition pour que s'établisse un plan limite d'expérience qui doit s'éprouver viscéralement. La perception bascule en mode nocturne. C'est dans un état légèrement modifié de conscience que l'on arpente alors l'espace à l'aveugle, toutes les autres formes de perception (auditive, olfactive, etc.) se trouvant décuplées. Les fantômes ont une odeur, ils aspirent, dit-on, l'énergie autour d'eux, un mécanisme qui se manifeste par des chutes brutales de température, parfois 


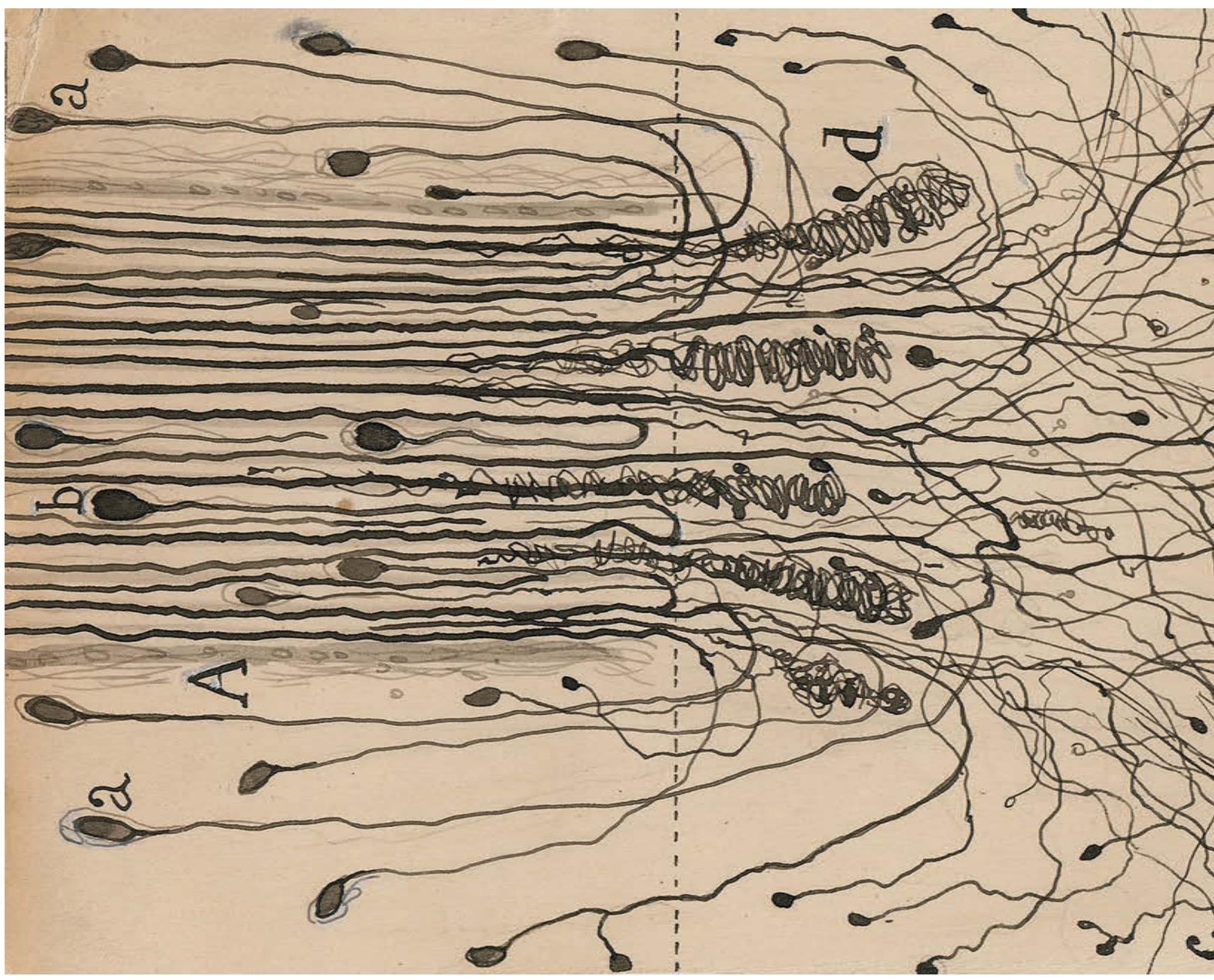

à même la peau du chasseur, qui se doit de garder les pores ouverts au moindre signal. II n'y a pas de présences pour qui ne sait rester réceptif, écouter et s'écouter, les sens exacerbés, attentif à la caisse de résonance que constitue son propre corps, en état d'hypervigilance. Nous avons filmé de nombreuses chasses. Difficile de ne pas éprouver une forme d'inquiétude, contagieuse et physique. Navigant sur un terrain autrefois habité, peut-être visité des milliers de fois, un espace potentiel plus qu'une réalité parallèle, les ghost hunters se rendent disponibles à tout un champ de possibilités spectrales paradoxalement très ancrées, suintant à même les murs, dans leurs aspérités.

Ils n'ont pas simplement besoin d'obscurité et de silence. II leur faut aussi un environnement exempt de toute perturbation électromagnétique, car c'est l'anomalie même dans ce champ qu'ils guettent, ou ce que Philip $K$. Dick appelle dans L'Exégèse la quantité sourde, une perturbation inexplicable dans le comportement de la réalité. Le bruit de fond permanent d'une ville 


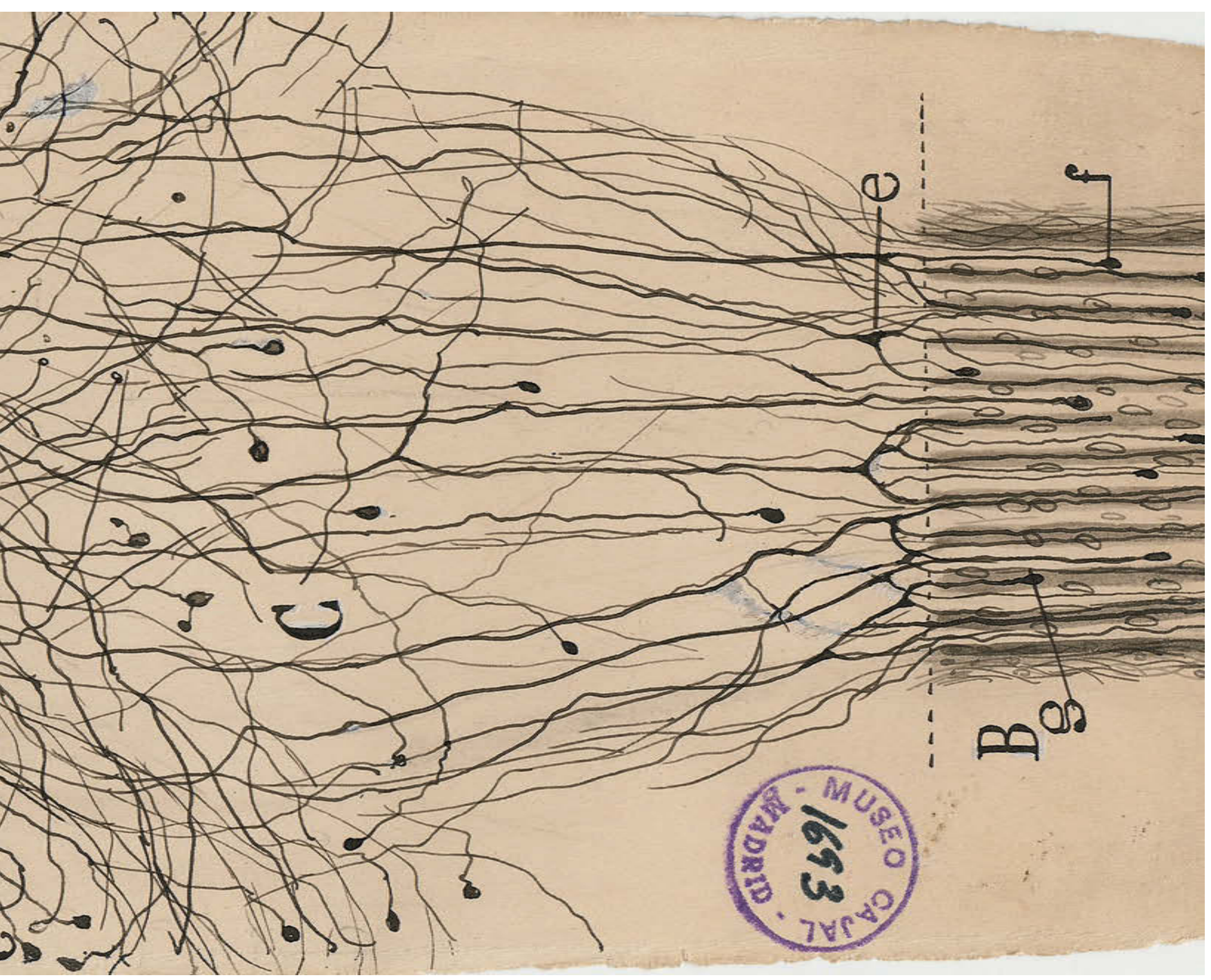

comme Calcutta rendant cette connexion profonde de plus en plus délicate à instaurer, ils n'hésitaient pas à aller voir ailleurs. Leur passion pour les lieux historiques et tout ce qui touchait à la période coloniale les poussa à organiser une expédition dans le Bihar, loin de tout opérateur wifi, à Simultala, une ancienne station de villégiature pour l'élite britannique de Calcutta. À l'intérieur d'un fort anglais sur lequel circulaient les pires rumeurs, où massacres et viols auraient été commis, à tel point que personne ne voulait y mettre les pieds à la nuit tombée, les ghost hunters détectèrent un champ magnétique inhabituel grâce au K2 Meter. Ils y revenaient régulièrement et finirent par localiser un point précis où le champ magnétique correspondait à la hauteur d'un être humain de taille moyenne. «1,64 m, une taille humaine! » s'exclama Soumen.

En ghost hunting, il s'agit moins de détecter purement et simplement des présences que de les piéger pour ensuite les faire parler. II y a donc deux phases bien distinctes: détection (ici au K2 Meter) et communication fig. 6

Santiago Ramón y Cajal, Vue de cellules nerveuses, 1913 (C) CSIC, droits réservés. 
14. Sur les fortunes de l'électromagnétisme en Inde et ses hybridations ayurvédiques, voir Mukharj 2016. L'auteur montre par ailleurs comment l'introduction de technologies dans le diagnostic des médecins ayurvédiques aux $x \mathrm{X}^{\mathrm{e}}$ et $\mathrm{X} \mathrm{x}^{\mathrm{e}}$ siècles à Calcutta a produit des transformations majeures dans les représentations du corps humain. Notre enquête va dans ce sens en montrant que l'usage de technologies de mesure magnétiques transforme radicalement les représentations du corps des fantômes, devenus des champs énergétiques, en Inde comme ailleurs Tout laisse à penser que les fantômes quantiques, qui n'en sont qu'à leurs balbutiements, en sont le prochain stade.

15. Alfred Binet a voulu tester le rapport aux stimuli, au signal et à son extrapolation dans I'hallucination, en mettant des filtres optiques sur les gens sujets à des apparitions fréquentes. Le fait que les fantômes apparaissent déformés par ces filtres était la preuve selon Binet qu'il s'agissait d'extrapolations

à partir d'indices qui étaient dans la réalité. Et la conclusion de Binet était sans appel: la perception ordinaire est par essence un mécanisme d'extrapolation, tout juste poussé un peu plus en intensité dans ce qu'on appelle hallucination (Binet 1884 ; Binet et Feré 1885).
16. Parmi les critiques le plus souvent adressées au K2, il est dit que l'appareil peut détecter les fréquences extrêmement basses (50 à $1000 \mathrm{~Hz}$ ) et très basses (1 000 à $20000 \mathrm{~Hz}$ ), mais qu'il ne mesure qu'une petite partie du spectre. L K2 est aussi très sensible aux fluctuations des champs

électromagnétiques. Il est facile de recourir à des «trucs" pour faire s'allumer le K2, d'où son utilisation dans beaucoup d'émissions de télé pour reproduire des phénomènes de présence sur commande. (à l'Echovox ou à la Ghost Box). L'incroyable profusion de machines dans ce domaine facilite-t-elle vraiment la tâche ou n'est-elle qu'un leurre? Faut-il accorder aux machines elles-mêmes des capacités hallucinatoires? À partir du moment où les ghost hunters choisissent d'enquêter via des appareils, ils s'interrogent nécessairement à la fois sur ce que la technique peut ou non capter et sur ce que perçoivent les humains, confrontés aux angles morts de leurs propres sensations. Il y a ce que l'humain ne perçoit pas et pense que l'appareil capte, et il y a ce que l'humain pense percevoir que l'appareil ne perçoit pas. La chasse aux présences entraîne ainsi le ghost hunter dans un espace peuplé d'ondes ${ }^{\mathbf{1 4}}$, en quête d'une fréquence où la communication sera possible, ce qui n'a au premier abord rien d'humain.

Suspendus au signal émis par le K2 Meter, les chasseurs peuvent facilement succomber à la tentation d'anthropomorphiser ce à quoi ils ont affaire, le désir et l'attente jouant ici un rôle prépondérant. Dès qu'un signal est pressenti, quelque chose de l'ordre d'un pacte hallucinatoire se met en branle. Celui-ci, ne nous méprenons pas, n'a rien à voir avec l'imagination du cerveau des ghost hunters. S'il y a là un mécanisme hallucinatoire ${ }^{\mathbf{1 5}}$, il est peut-être avant tout dans la machine elle-même, qui traduit un signal faible reçu en signal fort, bel et bien visible grâce à un voyant lumineux. Le succès de tels appareils tient aux possibilités multiples de variation du contrat sensoriel qu'on peut sceller avec eux. En effet: «Qui sent? », «Qui extrapole? »; il n'y a pas de réponse simple à ces questions. Ce que l'on cherche, c'est à établir une connexion avec un bruit présignalétique, un genre de bruit blanc ou de soupe quantique, d'avant les signaux, permettant potentiellement d'établir une communication et de recevoir des informations surnormales. Or le désir de trouver cette fréquence est vite rattrapé par celui d'entrer en contact avec un produit dérivé de présence humaine avec lequel une conversation normale serait possible. Ce désir est tel qu'on va poser au K2 des questions sur un mode oraculaire ${ }^{16}$. Questions auxquelles il ne pourra répondre que par oui ou par non: «Es-tu un homme?», «Es-tu une femme?», «Es-tu anglais?», «s-tu indien? », etc.

Leur impression se confirmant, les ghost hunters déballèrent dans le fort d'autres appareils. Notamment une Echovox et une Spirit Box, qui brouillent les champs radio: un peu comme lorsqu'on passe à toute vitesse d'une fréquence à l'autre, on n'entendait qu'une bouillie de phonèmes. Ils se mirent alors à poser toute une série de questions dans l'attente d'une réponse synchrone. Ce type d'appareils est la cible des critiques des chrétiens évangélistes, convaincus que la technologie ne fait qu'attirer les démons, mais aussi chez les ghost hunters eux-mêmes, de la part de ceux qui militent pour un ghost hunting sans instrument, reposant sur les seules facultés parapsychiques des humains. La plupart invoquent la paréidolie, ce phénomène psychologique qui consiste, à partir d'un stimulus, à extrapoler inconsciemment une forme familière dans un paysage, un nuage, de la fumée ou encore une tache d'encre. Mais cette critique ignore que les adeptes de l'Echovox, conscients du problème, se mettent à plusieurs pour éviter au maximum d'être leurrés par un seul cerveau. L'accusation de paréidolie n'a jamais mis fin à leur quête, et s'ils tiennent autant à faire l'expérience en groupe, c'est pour aller au-delà de la paréidolie, en franchissant méthodiquement des paliers successifs: d'abord, le seuil de présence au-delà duquel ils se disent qu'ils ont bien affaire à quelqu'un, puis celui de la paréidolie au-delà duquel 
ils se persuadent qu'ils ne sont pas en train d'halluciner, mais que quelque chose se communique à eux, et enfin le seuil de synchronicité dans les questions-réponses qui, s'il est franchi, leur fait éprouver le sentiment d'une connexion durable et non fugace. Ce sentiment de connexion est la grande émotion recherchée.

II n'y a donc pas de réponse simple à la question de savoir qui parle à travers l'Echovox, surtout quand l'expérience est faite en groupe. S'il était trop facile de se leurrer, l'exercice n'aurait pas grand intérêt. Une congruence entre la question et la réponse est assez rare. Seules des synchronicités durables dans le chaos des phonèmes permet de les attribuer à un esprit. Comment expliquer ces congruences? En fait, beaucoup des usagers de l'Echovox savent très bien qu'il y a une banque aléatoire de phonèmes à l'intérieur, mais ils la considèrent comme manipulable à souhait par des entités. Si tout appareil préchargé avec des extraits de son, qui fonctionnerait sur un mode aléatoire et jouerait des fichiers dans le désordre, génère une bande piratable par n'importe quelle entité parasite, rien n'empêche de penser que les esprits qui se manifestent ainsi pourraient accéder aux probabilités futures comme aux faits historiques, et ainsi créer de l'ordre à partir d'une bouillie aléatoire. Soumen en était convaincu: «Les appareils qui utilisent des fréquences magnétiques ou des ondes sonores peuvent être manipulés à des niveaux supérieurs de réalité, hors des capacités humaines. ”

Les ghost hunters sont face à la soupe d'ondes tels des hackers de l'invisible qui chercheraient le code d'accès. Tout comme les entités qu'ils tentent d'attirer puisqu'elles ont la capacité de parasiter les ondes. C'est même leur premier moyen d'expression. L'appareil est ainsi moins utilisé pour détecter que pour appâter des présences, au croisement des bandes passantes, entre plusieurs fréquences. En soutenant que les fantômes sont des genres de concrétions atomiques, les ghost hunters se mettent euxmêmes en position de pirater les répertoires de fantômes existants, mais pas uniquement. En effet, quand une voix bengalie s'exprime dans une soupe de mots anglais émise par une Echovox importée tout droit des États-Unis, il est clair que cette dernière a moins été conçue pour faire parler les fantômes bengalis que ceux de la guerre de Sécession! C'est toute une technologie made in USA qui se trouve détournée au profit de l'effort remarquablement méthodique d'occuper un angle mort de la perception humaine, mais aussi un angle mort de la science, de la technique et du langage.

Ce jour-là, dans le fort abandonné, les questions traduisaient bien le type de personne que les ghost hunters recherchaient sur les ondes: «Es-tu anglais ou indien? », «Es-tu mort de façon violente? », «As-tu tué des gens? », «Étais-tu un bourreau? », etc. Malheureusement, cette fois, ils n'obtinrent pas de réponse évidente, mais à la fin de l'interrogatoire, ils demandèrent à la présence si elle souhaitait continuer à parler ou les voir partir. Le signal émis fut pour une fois d'une grande clarté. Le K2 se mit à clignoter intensément et nos amis en conclurent qu'il valait mieux déguerpir immédiatement. Beaucoup de leurs questions auraient très bien pu être posées par Trupti aux hypnotisés impliqués dans l'Attentat du général anglais. À des lieux les uns des autres, tous semblaient évoluer dans un même espace de synchronicités. Mais Trupti était déjà ailleurs. Pendant que les ghost hunters terminaient leurs 
17. Il est dit très peu de choses du contenu de la mémoire génétique dans les travaux de biologie moléculaire sur la mémoire (Squire et Kandel 2002). Damasio ne consacre qu'une note très brève à l'inconscient génomique (2012).

18. L'équivalence entre mémoire et imagination est l'un des arguments forts de L'Autre Moi-même (Damasio 2012).

\footnotetext{
ci-contre

fig. 7

Santiago Ramón y Cajal, Cellules nerveuses, s.d. akg-images/Science Source.
}

investigations à Simultala, elle avait mis sous hypnose plusieurs personnes pour qui la Lutte pour l'indépendance n'était qu'une couche intermédiaire dans leur existence, mais sûrement pas la dernière. On pouvait aller bien plus profond dans le piratage de l'invisible.

\section{Synchronicité}

Trupti commençait à entrevoir certaines régularités propres au biotope de Calcutta. Nombreux étaient les hommes qui s'imaginaient avoir été sâdhu dans leurs vies antérieures, à deux doigts de sortir enfin du cycle des naissances, mais par un accident ou un bug, ils avaient été propulsés dans leur corps actuel et se demandaient quelle pouvait bien être leur mission. «Pourquoi es-tu revenu? » demanda Trupti à l'un d'entre eux, qui répondit: «Unfinished business. " De nombreuses femmes, quant à elles, se retrouvaient en combattantes de l'indépendance. Plusieurs explications peuvent être invoquées, en dehors de l'idée qu'on a vraiment affaire à des «vies antérieures ». Soit l'imagination des hypnotisés est limitée, et il y a autant de chances pour un habitant de Calcutta de se retrouver sous hypnose en pleine lutte pour l'indépendance ou en sâdhu que pour un Parisien sous hypnose d'avoir joué un rôle dans la Révolution française, participé à la prise de la Bastille ou vécu sous Napoléon. Soit la mémoire collective coagule en chacun selon des chemins que l'on ne soupçonne pas, et des personnages et épisodes oubliés pourraient bien se manifester au moment où l'on s'y attend le moins, si comme le disait Kalyan, «quelque chose est stocké quelque part».

Tout un corpus rituel depuis l'Inde védique est d'ailleurs fondé sur le postulat que l'embryon se souvient de tout mais perd la mémoire au moment de la naissance. "J'ai vu des milliers de matrices, j'ai mangé tant de nourritures diverses, bu à tant de seins. Je suis né dans 84 millions de ventres, mort et rené encore. À chaque naissance, au contact de l'air, la mémoire des vies et des morts passées, des bonnes et mauvaises actions et de tout ce qui leur est lié, s'évanouit. » Ainsi parle l'embryon, doté d'une mémoire pleine, dans le Garbhopanishad (Traité de l'embryon, -2000 av. J.-C.). Un embryon qui se souviendrait de tout et perdrait sa faculté mnésique à la naissance, voilà une belle hypothèse contre-intuitive qui entre en résonance avec les débats les plus contemporains sur les frontières de la mémoire (génétique, génomique, cellulaire, etc.) ${ }^{\mathbf{1 7}}$. Et si l'hypothèse la plus contre-intuitive était aussi la plus vivante? Elle a le pouvoir de stimuler enquêtes, expériences, protocoles. Et on ne doit pas s'étonner que la possibilité de la réincarnation s'ancre toujours dans une anomalie mnésique, une impression de déjà-vu, une ressemblance curieuse, tous ces indices sans lesquels elle ne pourrait s'épanouir comme virtualité. Si la réincarnation survit ainsi à l'état spectral, alors qu'elle a été régulièrement soumise au tribunal scientiste de la preuve, c'est qu'elle est aussi ce que nous en faisons, un plan limite d'expérience, ce que Trupti avait bien compris, la mémoire et l'imagination constituant sous hypnose une seule et même chose ${ }^{\mathbf{1 8}}$. Et le fait que religieux, scientifiques et chercheurs amateurs se risquent à mettre la réincarnation sur le terrain expérimental a au moins le mérite d'éviter sa sclérose dans un dogme, dans une croyance figée, ou sa relégation du côté des hypothèses mortes. 


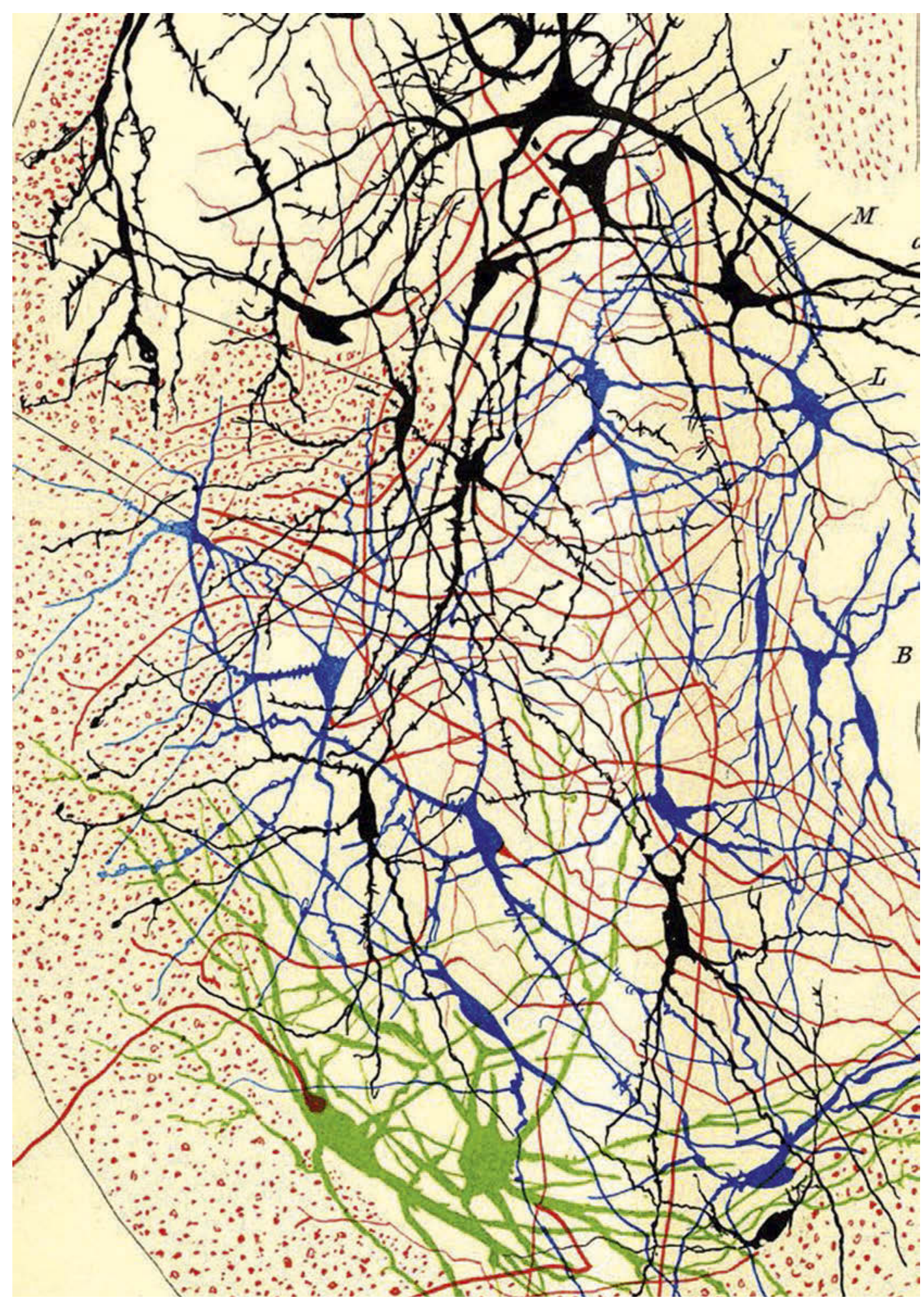


19. Henri Friedel désigne ainsi non pas un monde flottant séparé de la vie, mais tout ce qui meurt au sein de la pellicule terrestre et entre dans des processus écologiques de recomposition: "La thanatosphère a commencé d'exister en même temps que la vie et elle n'a jamais cessé d'augmenter ", dit-il (1980: 210)...
Les protocoles d'expérience les plus fascinants seraient alors ceux qui amènent à éprouver le milieu spectral comme un circuit étranger, autrement mental, une mécanique non humaine, un alien mind au sens de Dick. Là où le ghost hunting est une grande affaire de parasitage des ondes, I'hypnose ouvre une brèche comparable: les hypnotisés piratent l'histoire autant qu'ils se laissent parasiter par des personnages incongrus. Au fur et à mesure, Trupti mettait le doigt sur une formule nouvelle, à mi-chemin entre l'hypnose individuelle et la thérapie de groupe. S'il est possible de mettre plusieurs personnes sous hypnose dans la même pièce et de les embarquer dans une vision commune, l'Attentat du général anglais et les tentatives de connexion des ghost hunters avec des personnages historiques démontraient autre chose: rien de mieux qu'une sélection de sujets et/ou de lieux à l'aveugle pour piéger fantômes et réincarnés évoluant dans des recoins apparemment distants de la «thanatosphère 19 ».

Dans le cabinet de Trupti, un nouveau motif commun aux récits d'hypnose surgit le jour où Hiran, un artiste bengali, la consulta pour une phobie de l'eau. Hiran avait failli se noyer plusieurs années auparavant en tombant d'un ferry et avait été miraculeusement sauvé par des pêcheurs. Trupti, bien décidée à régler son problème, l'amena à revivre son traumatisme par une induction où elle l'embarqua sur un bateau en pleine tornade. Elle fit de même avec d'autres hypnotisés qui se présentèrent à elle avec des problèmes similaires. Tous montent sur un même bateau à Calcutta, direction Venise, en quête de nouveaux horizons. Quand Trupti immergea Hiran à nouveau au fond des eaux, il y rencontra cette fois une superbe créature et fut rempli d'un sentiment d'unité jamais éprouvé. Après s'être mué en serpent, il fit l'amour avec un arbre (réincarnation de la femme rencontrée précédemment). D'autres ne furent pas aussi chanceux. Siddharth, un pilote de la Merchant Navy, soupçonnait une anomalie dans son karma derrière ses échecs amoureux à répétition. Après l'avoir mis sur un bateau, Trupti déclencha la tornade et Siddharth fut propulsé au Moyen Âge dans le corps d'un gladiateur sanguinaire qui tua femmes et enfants, mais aussi le mari de la femme qu'il convoitait et qui ressemblait étrangement à sa compagne actuelle, une jeune Ukrainienne qu'il avait connue sur un site de rencontres. II était clair pour lui que s'il l'avait choisie, c'était pour expier tout le mal qu'il avait commis. Sanchita, une danseuse d'odissi, se retrouva pour sa part à l'époque médiévale, danseuse de temple au IX ${ }^{\mathrm{e}}$ siècle, noyée une première fois par un garde royal qui cherchait à la séduire et qu'elle avait humilié, avant de se réincarner en cygne et de mourir noyée à nouveau après avoir avalé un objet non identifié. Là encore, Trupti ne put rien faire pour la sauver. II y a des hypnoses destinées à finir au fond des mers. Debashish, le juge qui faisait du ghost hunting sur son temps libre, se retrouva dans le corps d'un peintre nommé Charles Donatella, qui fuit Calcutta par la mer et périt dans un naufrage après avoir fait un détour par Venise. Trupti, désemparée, n'arriva pas à le sortir de là et il se réveilla de son hypnose avec une migraine épouvantable. Quant à Satarupa, qui consulta Trupti pour une phobie du sang, elle avait fui le Bengale suite à l'assassinat de son mari et avait cherché à tout oublier sous une autre identité, celle d'Elena: elle vécut une idylle avec un peintre en Italie, avant de se retrouver prise dans de sanglantes émeutes politiques à Rome et de mourir à nouveau, cette fois de tuberculose. Le seul à s'en être tiré 
véritablement fut Kanai Das Baul, un barde aveugle, qui passait son temps à rire sous prétexte qu'il connaissait très bien ses vies antérieures, qu'il avait déjà explorées avec son gourou, et que l'exercice ne faisait pas grand sens pour lui. II expliqua à Trupti les raisons de sa cécité: il s'était crevé les yeux après avoir trop lorgné les femmes. II prit ensuite son instrument à corde (ektarā) et entonna une chanson nous rappelant qu'on ne peut contrôler la mort et qu'il vaudrait mieux écouter les bardes qui disent que la vie est un grand mirage (mayajāl).

Cette propension à la noyade chez les hypnotisés de Calcutta surprit Trupti. Au montage, le motif s'imposa comme une deuxième bande passante permettant, à défaut d'une synchronisation totale des hypnoses les unes avec autres, un simple embranchement. II ne fallait pas s'attendre à une synchronicité forte sur toute une chaîne événementielle comme dans l'Attentat du général anglais. En revanche, une synchronicité lâche ou accidentelle sur le mode d'un partage d'univers n'était pas absurde. Et si tous s'étaient retrouvés sur le même bateau, piloté par le sâdhu aveugle, sans en connaître la véritable destination? Voilà bien le seul moyen plausible d'imbriquer des récits aussi hétérogènes: un naufrage en mer piloté par un sâdhu en disait plus long que chaque naufrage individuel, sans que Trupti ne sache vraiment quoi. Pour elle, le plus inquiétant était son impuissance à faire bifurquer l'hypnose, comme habituellement, pour que le patient dépasse sa propre mort et retrouve le goût de la vie dans un autre corps. Était-ce le signe de sa propre déroute ou bien, comme dans l'Attentat contre le général anglais, qu'il fallait aller encore plus profond? L'hypnose de Kiran amorçait le naufrage, Debashish le clôturait. Et entre les deux, les personnages se succédaient en une longue chaîne médiumnique guidée par le principe suivant: je suis dans ton hypnose, tu es dans la mienne et le dernier à faire progresser le récit décidera de notre sort.

\section{Loterie cosmique}

Parmi les patients qu'il fut donné à Trupti d'hypnotiser, l'un se démarqua par l'intensité de sa vision. Parfois victime de vertiges, Joy était astrologue. Il avait consulté un service de neurologie plusieurs fois depuis l'enfance, cependant les IRM n'indiquaient rien d'anormal. Trupti lui demanda s'il avait déjà fait une séance d'hypnose. II répondit que non, mais à peine le mot d'hypnose prononcé, Joy entra dans une transe profonde, les yeux révulsés, et sa voix se modifia radicalement. Trupti tenta plusieurs fois de le réveiller pour l'asseoir plus confortablement, mais sans succès. La vision riche en couleurs de Joy est sans doute la plus spectaculaire à laquelle nous avons assisté. Cette fois, l'étonnement ne venait pas d'un circuit partagé de synchronicités que Trupti aurait par mégarde éveillé sans jamais être en mesure d'en cerner les contours, mais plutôt du flot énigmatique des images qui s'empara de son patient. Trupti se contenta de jouer son rôle de machiniste d'un cinéma mental, tandis que Joy décrivait la courbe étonnamment précise de ses incarnations. Assistant du Bouddha en -600 av. J.-C., il avait été lotus, perdu quelque part dans l'actuel Canada à l'aube de l'humanité, puis vécut toute une série de réincarnations tragiques: torturé comme esclave noir, mort brûlé dans son champ alors qu'il était paysan dans le sud de l'Inde et s'appelait 


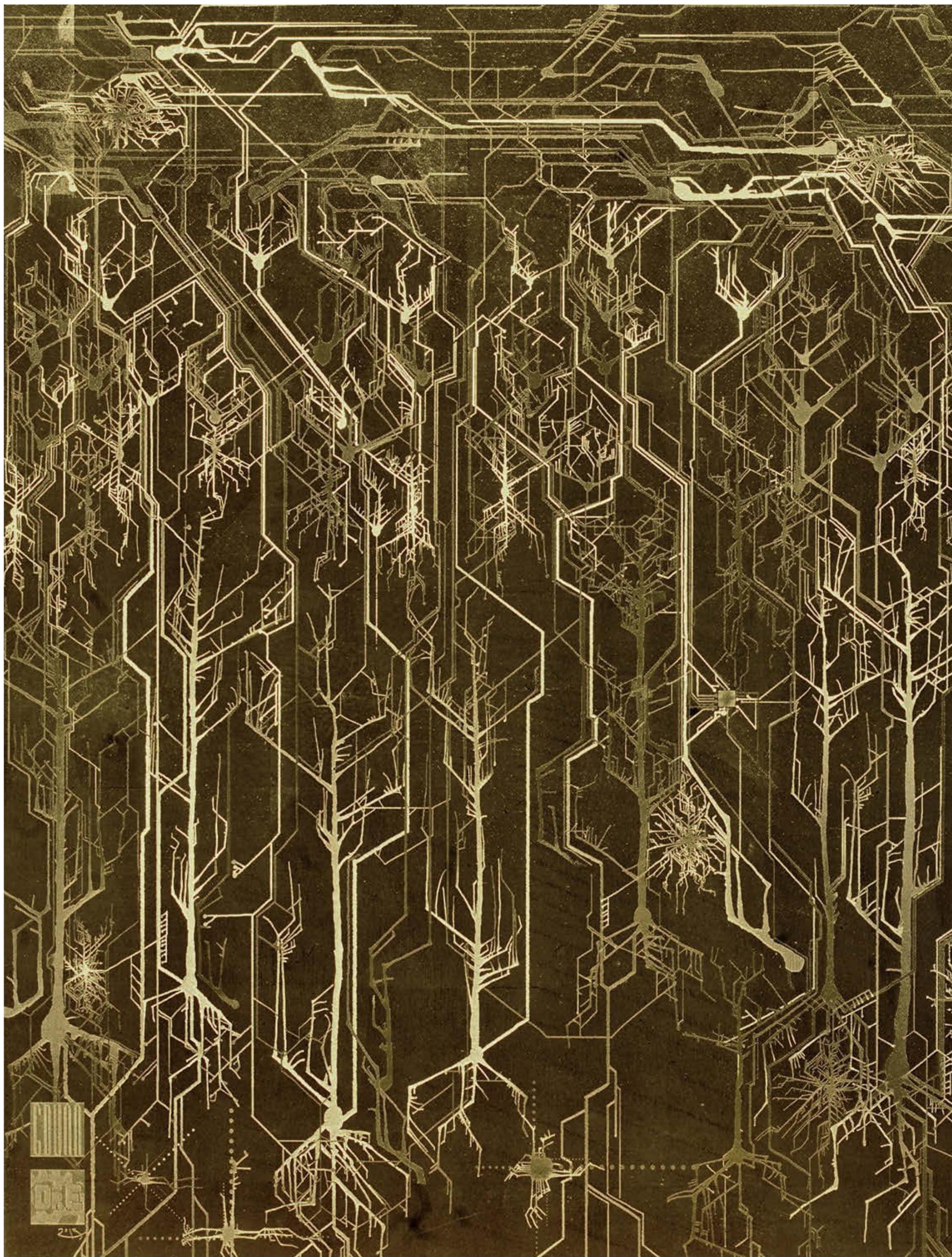




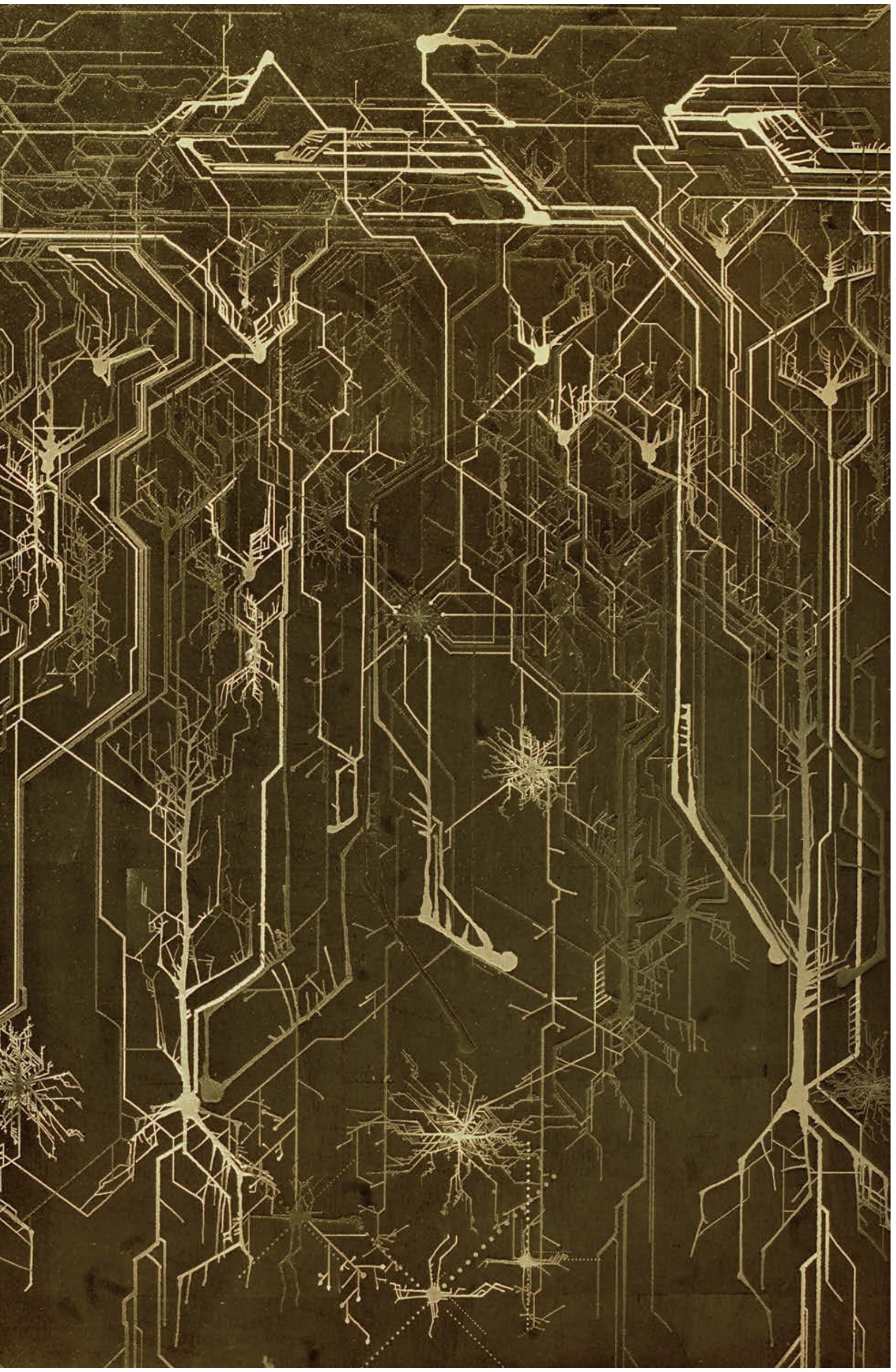


Murugan, il endura de terribles souffrances avant de vivre une série d'incarnations animales (paon, nymphe, etc.), mais pour des raisons à chaque fois différentes, il échoua dans sa libération et revint sous une nouvelle forme. Qu'un lotus se retrouve bodhisattva (Éveillé) au vle siècle est une progression assez compréhensible, mais un esclave noir torturé, un paon capable de voir et de sentir des choses que d'autres ne voient pas, une pure lumière au royaume des nymphes (Gandharva Loka) avant de se retrouver dans son corps à lui? L'hypnotiseuse eut bien du mal à savoir s'il pourrait retenir une leçon quelconque de ces incarnations successives, excepté le fait qu'il lui restait vingtcinq ans à vivre avant de rejoindre Bouddha à nouveau. S'il y avait une logique dans cette destinée, elle semblait aberrante, rebelle à toute progression. Joy revint cependant de ce voyage avec une information décisive: il lui manquait encore le secret de l'immortalité que le Bouddha ne lui avait pas livré, bien qu'il eût été l'un de ses plus proches disciples. Autrement dit, Joy avait approché de très près sa libération du cycle des naissances, mais avait échoué, y retombant aussitôt. Et Bouddha ne lui légua aucun message à transmettre. «À quoi bon? » dit Joy, laissant Trupti très perplexe. « J'ai toujours cherché à être pur dans ma forme», dit-il. Puis il ajouta: «Je n'ai jamais été une rose. " Ainsi Joy achevait son voyage sur un sentiment d'incomplétude, un état du vivant qui n'avait jamais été exploré. Quand il se réveilla, il avait tout oublié et ne voulut jamais recommencer l'exercice. Grâce à lui pourtant, Trupti mit le doigt sur une propriété inconnue du circuit spectral. S'il est tentant de penser que sa prochaine réincarnation est déterminée par ses karma antérieurs, personne ne peut prédire s'il sera une grenouille ou un commerçant. Joy avait vécu dans sa chair l'aberration du samsâra. Peut-on espérer, par l'hypnose, se faire une idée plus précise de sa cybernétique? À force de jouer à transmigrer et simuler la sortie hors de son corps, de nouvelles possibilités se faisaient jour. L'hypnose révélait à chacun l'étonnante singularité de sa destinée, sa courbe capricieuse, une parmi tant d'autres possibles.

Les ghost hunters, poursuivant leur enquête sur les rives du Gange, eurent une révélation comparable en intensité à l'hypnose de Joy. Que faisaientils là, à examiner des offrandes de rituels funéraires (tarpan) fraîchement déposées? Leur K2 Meter se mit soudain à clignoter. Même le pujari, persuadé que les rituels ne sont faits que pour rassurer les vivants, n'aurait pas cru à un tel scénario! Une communication d'une rare richesse fut établie via l'Echovox. Le rêve de Soumen de pouvoir mettre enfin une âme errante sur écoute semblait s'exaucer. «Je pense qu'une énergie intelligente est ici!» se dit-il, tout excité. Dans un flot de radiofréquences extrêmement perturbé, une bouillie de grésillements sonores, les ghost hunters tentèrent d'obtenir des réponses à leurs questions. Et s'ils étaient les seuls à les entendre, c'est peut-être qu'ils étaient aussi les seuls à prendre la peine de les écouter. Cette communication fut, d'après eux, l'une de leurs expériences de contact les plus spectaculaires et convaincantes des deux dernières années. La fréquence avait tenu suffisamment longtemps dans le chaos des interférences pour que s'établisse une connexion profonde, intime et durable avec un signal à peine audible. S'il fallait attendre avant de se prononcer, analyser la bande enregistrée à tête reposée, ils avaient réussi à deviner quelques noms, humer une odeur de friandises et éprouver dans leur chair la souffrance d'une âme qui les appelait à l'aide. Avec quoi les ghost hunters étaient-ils entrés en communication? Avec «une volonté de dire quelque chose que le mécanisme

\author{
Greg Dunn, Cortical \\ Circuitboard Gold, 2017 \\ (C) Greg Dunn. \\ double page \\ précédente \\ fig. 8
}


serait impuissant à reproduire», comme dirait James (1869: 212)? Ou avec une «énergie plasmatique», comme Dick s'était plu à l'imaginer, «un champ faible, niveau légumes » ou encore un cerveau cosmique mal irrigué? Pour nos investigateurs, il y avait d'autres hypothèses à explorer en cet endroit du biotope, un circuit à la fois plus local et plus alambiqué. La voix s'appelait Shishir, rosée en bengali, un prénom très courant, autant probablement que ne l'était le sentiment d'incomplétude éprouvé par Joy de n'avoir jamais été une rose. Shishir était mort de façon traumatique et aimait les friandises.

$\mathrm{Ni}$ Trupti ni les ghost hunters n'auraient pu imaginer un lien quelconque entre Joy et Shishir (parasitage, télépathie ou réincarnation?). Pourtant, il y a bien quelque chose de commun entre le cerveau de Joy et l'espace où évolue Shishir. Le premier fait des connexions synaptiques sur un mode frénétique et ce mental se révèle être sous hypnose un milieu chaotique où prospèrent les spectres, tandis que l'autre est un milieu perturbé qui s'avère, par le ghost hunting, contenir du mental qui nous échappe. Chez Joy, un incroyable flux d'images se produit, que l'hypnotisé commente en temps réel, et auquel Trupti n'a accès que de façon indirecte, par les mots de l'hypnotisé. Elle ne peut qu'entrevoir les bribes énigmatiques de son curieux destin, par étincelles accidentelles. Loin d'être réductible à du récit, l'intense activité neuronale, crépitante et chaotique, est palpable derrière chaque vibration oculaire, dans chaque battement de paupière. Joy est en proie à des éclairs mystiques et aux larmes. Sa révélation n'a rien à voir avec le sentiment de quiétude océanique décrit par les sciences de l'extase (Hulin 2008b). Des connexions synaptiques s'opèrent en temps réel dans un cerveau en surrégime. Et probablement que Trupti, qui avait eu tant de mal à mettre sous hypnose certains de ses patients (rappelons-nous M. Som), aurait pu dire de Joy ce que les ghost hunters ont conclu de leur communication avec Shishir: une fréquence s'est trouvée, une connexion durable s'est établie.

\section{Conclusion}

De quelle connexion s'agit-il? On espère avoir montré que l'hypnotiseuse fait tout pour atteindre avec ses patients un point de singularité, un seuil critique à partir duquel leurs vies «antérieures » vont se dévoiler. Et c'est aussi un seuil critique dans la dissolution des corps et leur rematérialisation que les ghost hunters cherchent à approcher ou du moins à élucider, en guettant la moindre synchronicité. Que les technoprophéties d'aujourd'hui jouent avec les immatériels d'hier - le transhumanisme n'est peut-être après tout qu'un réincarnationnisme, avec et par les machines - dépasse le cadre de cet article (Grimaud 2019), mais il faut avoir en tête ce déplacement pour saisir l'ingéniosité cybernétique avant la lettre du samsâra. Le brouillage des radiofréquences donnait autant l'impression aux ghost hunters d'être en prise avec un milieu vivant que le chaos des images mentales donnait à Trupti le sentiment de toucher par l'hypnose au fond abyssal de l'être et, par son biais, à plus dissimulé encore: une information manquante sur la nature de la singularité fantôme. Dans le milieu spectral, le chaos des distorsions domine. Sans lui, aucun effet de résonance n'est possible, que ce soit à travers les étincelles d'images historiquement signifiantes obtenues sous hypnose ou les voix qui se font audibles le temps d'un instant au milieu des parasites. On comprend dans ce contexte la fascination des ghost hunters et plus largement des chercheurs contemporains du paranormal pour la physique quantique: quoi de plus intrigant que l'émergence dans le chaos de congruences 


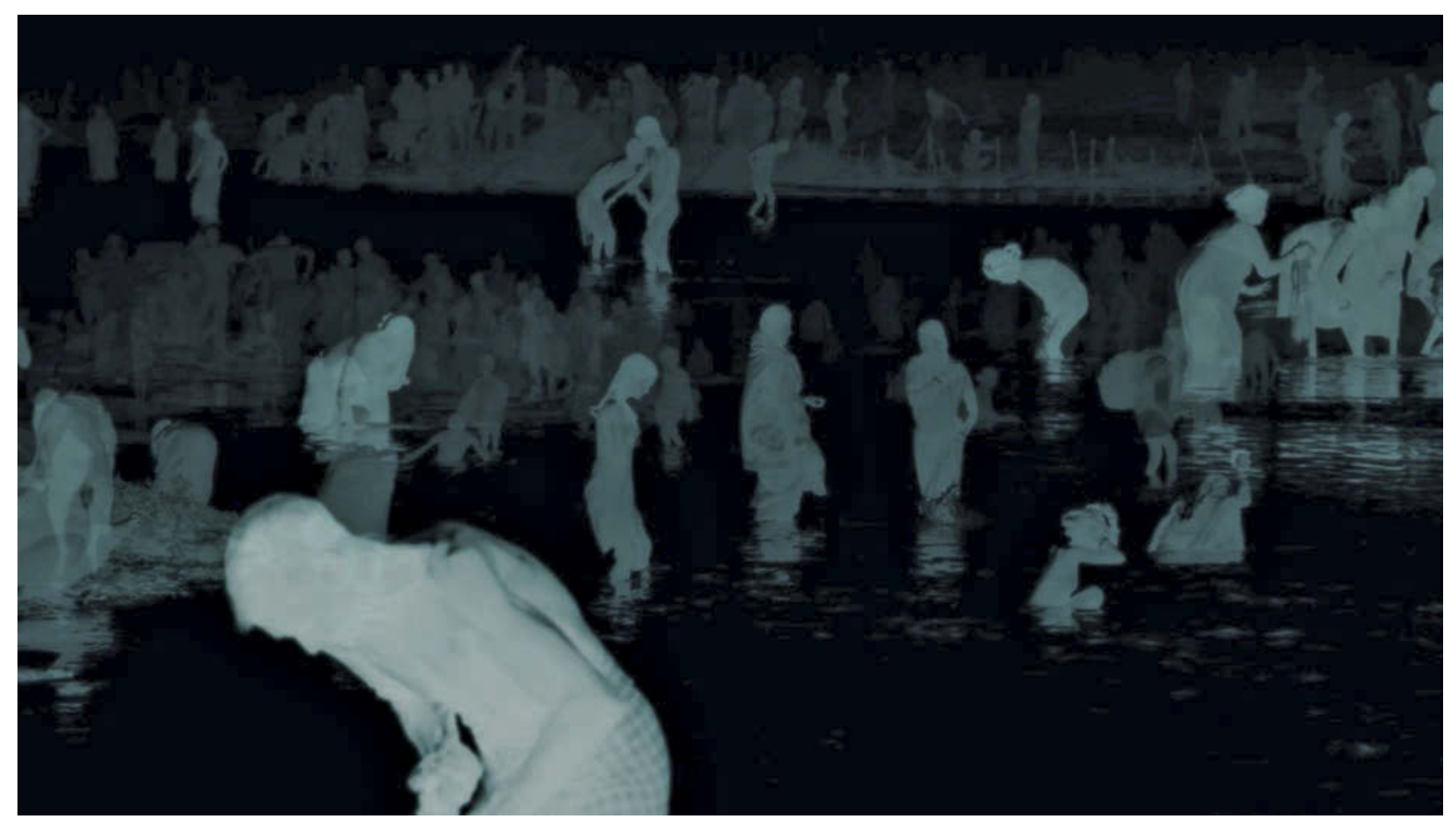


et de synchronicités! Tandis que la physique invoque le hasard et les probabilités, s'interdisant de passer du monde des particules à celui des personnes, les ghost hunters soupçonnent qu'il y a peut-être autre chose ici, à l'état corpusculaire; quelque chose d'assez humain pour s'adresser avec des mots à cette basse couche du réel, partie intégrante, quoique invisible, de notre propre monde. Joy a introduit, rappelons-nous, une nouvelle possibilité jamais envisagée auparavant par Trupti. Pour ceux qui vivent encore dans l'illusion que la transmigration est une machinerie bien huilée compilant et redistribuant les mérites karmiques ${ }^{\mathbf{2 0}}$, allant vers toujours plus d'amélioration, ou qu'il existe des règles claires qui décideront de votre prochaine réincarnation, il suffit de se remémorer Joy se débattant avec ses propres spectres pour envisager l'impensable: et si le samsâra n'était au fond qu'une grande loterie jouant avec les codes du vivant et remuant les possibilités aléatoirement? Une machinerie folle ou déréglée qui, contrairement à ce que voudraient nous faire croire ceux qui projettent dans la transmigration leurs derniers espoirs de progression, dessine des figures aberrantes ${ }^{21}$ ?

CNRS-LESC emmanuel.grimaud@gmail.com
20. Rappelons que contrairement aux approches théosophistes ou spirites, les versions pessimistes tendent à dominer l'Orient - le sujet doit tout faire dans I'hindouisme pour échapper au cycle des renaissances. Sur l'ancienneté de ce malentendu, voir : De Rochas 1911; et surtout Hulin 1985.

21. C'est le sentiment ambivalent qu'un dessein aberrant plutôt qu'intelligent (mais empreint de justice chrétienne) agite

souterrainement la réalité qui achève La Transmigration d'Archer de Dick: l'évêque Archer se réincarne dans le corps de Bill, un jeune attardé schizophrène jusque-là incapable de pensée abstraite, et soudain capable de s'exprimer dans un langage riche en métaphores et références bibliques. 
La singularité fantôme. Hypnose, chasse aux spectres et cybernétique de la transmigration à Calcutta Par Emmanuel Grimaud

Banks, Joe

2001 "Rorschach Audio: Ghost Voices and Perceptual Creativity", Leonardo Music Journal 11: 77-83

\section{Baudouin, Philippe}

2015 "Machines nécrophoniques introduction in Thomas Alva Edison [1948], Le Royaume de l'au-delà, trad. de l'anglais par Max Roth. Paris, Jérôme Millon: 7-81.

2018 «Archéologie des machines occultes ", Fantômes, Terrain 69: 96-113.

\section{Bernal, John Desmond}

1929 The World, the Flesh \& the Devil: An Enquiry into the Future of the Three Enemies of the Rational Soul. London, Kegan Paul, Trench, Trubner \& Co

\section{Binet, Alfred}

1884a «L'hallucination. I. Recherches théoriques ", Revue philosophique de la France et de l'étranger 17, avril : 377-412.

1884b « L'hallucination. II., Recherches expérimentales", Revue philosophique de la France et de l'étranger 17, mai : 473-502.

\section{Binet, Alfred et Féré, Charles}

1885 "La théorie physiologique de l'hallucination", Revue scientifique 35, 10 janvier : 49-53.

\section{Blanes, Ruy et Espirito} Santo, Diana (dir.)

2013 The Social Life of Spirits. Chicago, University of Chicago Press.

\section{Dick, Philip Kindred}

2006 [1982] La Trilogie divine, t. III : La Transmigration de Timothy Archer, trad. de l'anglais par Alain Dorémieux. Paris, Gallimard.

2016 [1974-1982] L'Exégèse, t. I, éd. par Pamela Jackson et Jonathan Lethem, trad. de l'anglais par Hélène Collon. Paris, J'ai Lu.

\section{Damasio, Antonio}

2010 L'Autre Moi-même les nouvelles cartes du cerveau, de la conscience et des émotions, trad. de l'anglais par Jean-Luc Fidel. Paris, Odile Jacob.

\section{Demiéville, Paul}

1987 [1952] Le Concile de Lhasa: une controverse sur le quiétisme entre bouddhistes de l'Inde et de la Chine au viiie siècle de l'ère chrétienne. Paris, Collège de France, Institut des hautes études chinoises.

\section{Friedel, Henri}

1980 Dictionnaire de l'écologie et de l'environnement.

Paris, Larousse.

\section{Goswami, Surendranath}

1911 "Bhut Bigyan »,

Janmabhumi 18 (3) : 126-27.

1914 Ayurved o Malaria-jwor. Calcutta, Janmabhumi Press.

\section{Grimaud, Emmanuel}

2016 «Renaître en temps réel. Expériences de régression de mémoire et vies antérieures ", Terrain 66: 24-45.

2019 «Silicium Prophétie.

La singularité technologique selon Vinge et le rapt de l'Apocalypse ",

Terrain 71: 140-157.

\section{Guenzi, Caterina} et D'Intino, Silvia

2016 "Un air de déjà-vu », Terrain 66 : 46-61.

\section{Hulin, Michel}

1983 "Corps de transmigration et corps de résurrection ", Eranos 52 : 347-388.

1985 La Face cachée du temps : l'imaginaire de l'au-delà. Paris, Fayard.

2008a Comment la philosophie indienne s'est-elle développée? La querelle brahmanes-bouddhistes. Paris, Éditions du Panama.

2008b La Mystique sauvage: aux antipodes de l'esprit. Paris, PUF.

\section{James, William}

2000 [1869] Expériences d'un psychiste, trad. de l'anglais par E. Durandeaud. Paris, Payot.

2005 [1897] La Volonté de croire, trad. de l'anglais par Loÿs Moulin. Paris, Empêcheurs de penser en rond.

\section{Kapani, Lakshmi \\ 1976 Garbhopanisad. \\ Paris, Adrien Maisonneuve.}

1993 La Notion de samskara dans l'Inde brahmanique et bouddhique. Paris, Collège de France/Institut de civilisation indienne/De Boccard.

Keyes, Charles F. et Daniel, Valentine E. (dir.)

1983 Karma: An Anthropological Inquiry. Berkeley, University of California Press.

\section{Luria, Alexandre}

1995 "Une prodigieuse mémoire», in L'Homme dont le monde volait en éclats, trad. du russe par Fabienne Mariengof et Nina Rausch de Traubenberg. Paris, Seuil.

\section{McGaugh, James}

2014 "Highly Superior Autobiographical Memory ", Scientific American, février 2014 40-45.

\section{Malamoud, Charles}

2004 «Un vent violent m'a séparé de moi ", L'Inactuel 10: 9-18.

\section{Mukharji, Projit Bihari}

2016 Doctoring Traditions: Ayurveda, Small Technologies and Braided Sciences. Chicago, University of Chicago Press.

Nees, Michael A. et Phillips, Charlotte

2014 "Auditory Pareidola: Effects of Contextual Priming on Perceptions of Purportedly Paranormal and Ambiguous Auditory Stimuli ", Applied Cognitive Psychology 29: 129-134.

\section{Raudive, Konstantin}

1971 Breakthrough: An Amazing Experiment in Electronic

Communication with the Dead

(The Inaudible Becomes Audible). New York, Taplinger.

\section{Rochas d'Aiglun, Albert (de)}

1911 Les Vies successives: documents pour l'étude de cette question. Paris, Bibliothèque Chacornac.

\section{Sconce, Jeffrey}

2000 Haunted Media: Electronic

Presence from Telegraphy

to Television. Durham,

Duke University Press.

Squire, Larry R. et Kandel, Eric R.

2002 La Mémoire: de l'esprit aux molécules, trad. de l'anglais par Béatrice Desgranges et

Francis Eustache. Paris, De Boeck.

\section{Stevenson, Ian}

2002 Réincarnation et biologie: la croisée des chemins.

Paris, Dervy.

\section{Tresch, John}

2011 «Experimental Ethics and the Science of the Meditative Brain ", in Francisco Ortega et Fernando Vidal (dir.), Neurocultures: Glimpses into an Expanding Universe.

Francfort, Peter Lang : 45-64.

\section{Vinge, Vernor}

2019 [1983] «Atteindre la Singularité », Terrain 71: 140-143.

1993 "The Coming Technological Singularity: How to Survive in the Post-Human Era ", Vision-21: Interdisciplinary Science and

Engineering in the Era of

Cyberspace, NASA CP 10129 :

11-22. page 50 et ci-contre Chasseurs de fantômes. Film Black Hole (détail) Emmanuel Grimaud, 2019 


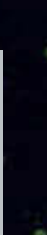

-

+ .

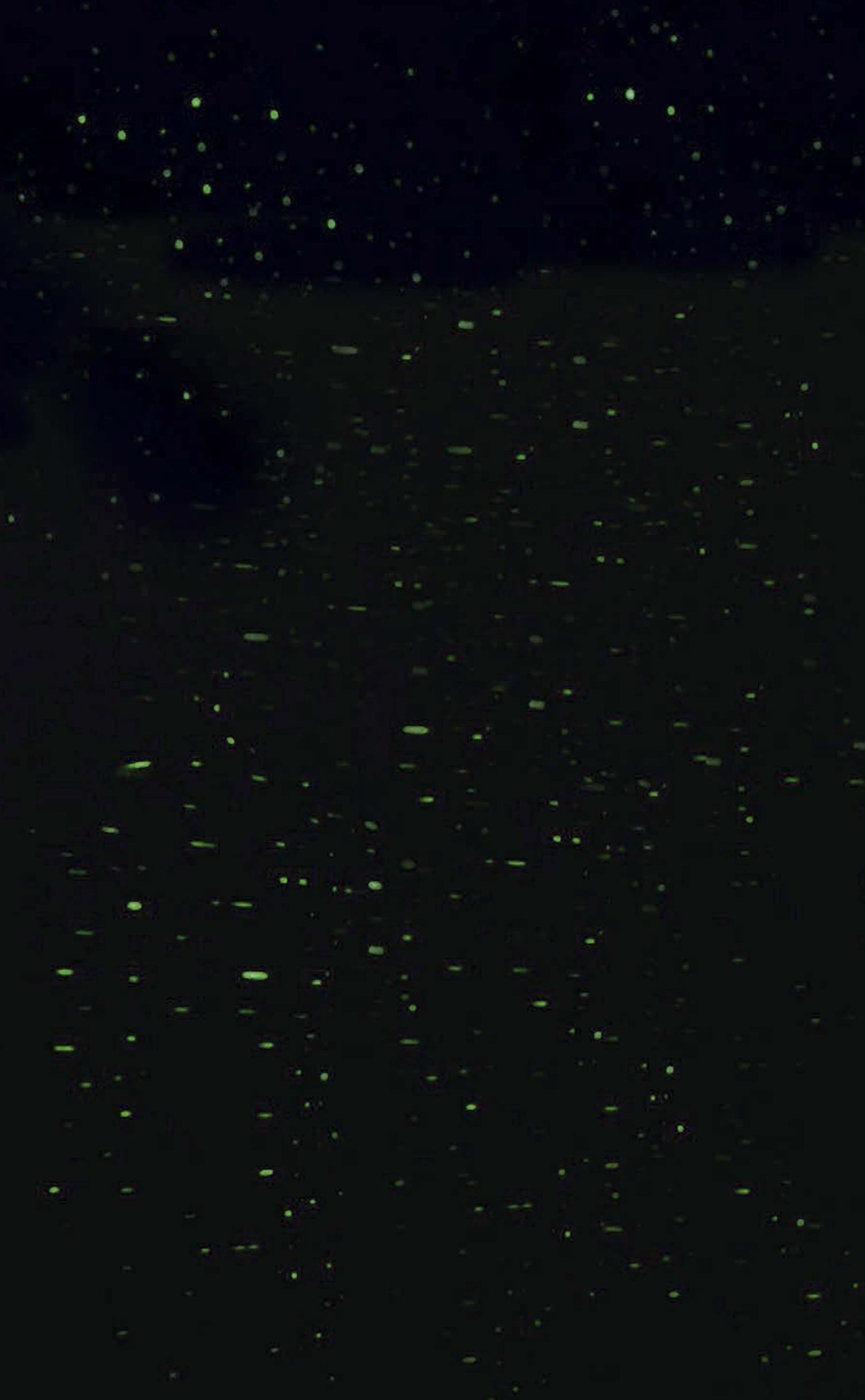

